\title{
Spin Needlets for Cosmic Microwave Background Polarization Data Analysis
}

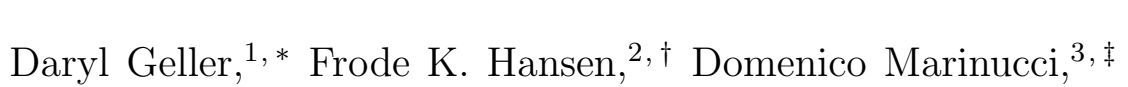 \\ Gerard Kerkyacharian, 4 , and Dominique Picard ${ }^{5,4}$ \\ ${ }^{1}$ Department of Mathematics, Stony Brook University, \\ Stony Brook, NY 11794-3651, USA \\ ${ }^{2}$ Institute of Theoretical Astrophysics, University of Oslo, \\ PO Box 1029 Blindern, N-0315 Oslo, Norway \\ ${ }^{3}$ Dipartimento di Matematica, Università di Roma 'Tor Vergata', \\ Via della Ricerca Scientifica 1, I-00133 Roma, Italy \\ ${ }^{4}$ Laboratoire de Probabilitè et Modèles Alèatoires \\ ${ }^{5}$ Universitè Paris 7 and Laboratoire de Probabilitè et Modèles Alèatoires
}

(Dated: March 6, 2022)

\begin{abstract}
Scalar wavelets have been used extensively in the analysis of Cosmic Microwave Background (CMB) temperature maps. Spin needlets are a new form of (spin) wavelets which were introduced in the mathematical literature by Geller and Marinucci (2008) as a tool for the analysis of spin random fields. Here we adopt the spin needlet approach for the analysis of CMB polarization measurements. The outcome of experiments measuring the polarization of the CMB are maps of the Stokes Q and U parameters which are spin 2 quantities. Here we discuss how to transform these spin 2 maps into spin 2 needlet coefficients and outline briefly how these coefficients can be used in the analysis of $\mathrm{CMB}$ polarization data. We review the most important properties of spin needlets, such as localization in pixel and harmonic space and asymptotic uncorrelation. We discuss several statistical applications, including the relation of angular power spectra to the needlet coefficients, testing for non-Gaussianity on polarization data, and reconstruction of the $E$ and $B$ scalar maps.
\end{abstract}

PACS numbers: 95.75.Mn, 95.75.Pq, 98.70.Vc, 98.80.Es, 42.25.Ja

${ }^{*}$ Electronic address: daryl@math.sunysb.edu

${ }^{\dagger}$ Electronic address: frodekh@astro.uio.no 
‡Electronic address: marinucc@mat.uniroma2.it

${ }^{\S}$ Electronic address: kerk@math.jussieu.fr

ฯElectronic address: picard@math.jussieu.fr 


\section{INTRODUCTION}

The WMAP satellite has provided the scientific community with the highest resolution full-sky data of the Cosmic Microwave Background (CMB) obtained to date ([1]). These data have allowed precise estimates of the temperature angular power spectrum $C_{\ell}$ up to the third Doppler peak and thereby high precision measurements of many cosmological parameters. In addition to measuring the temperature fluctuations in the CMB with high sensitivity, WMAP has also measured the polarization of the background radiation. But the polarization signal is almost an order of magnitude smaller than the temperature signal and is therefore measured with much lower sensitivity. The low signal-to-noise level makes it important to have a good understanding of systematic effects, in particular correlated noise properties. Little is so far known about polarized foregrounds. For low signal-to-noise data, small errors in the understanding of systematic effects and foregrounds could lead to errors in the estimates of the polarization angular power spectra.

In the near future, the situation with respect to CMB polarization will improve significantly. The Planck satellite will take full-sky measurements of the polarized CMB sky ([2]) and several other ground/balloon based experiments will follow up with high sensitivity observations in smaller regions of the sky (the CLOVER, QUIET and QUAD experiments to mention a few). Both ESA and NASA are planning high sensitivity full-sky satellite borne experiments within the next 10-20 years.

Polarization measurements allow for estimation of three more angular power spectra, the $C_{\ell}^{T E}$ power spectrum measuring the correlation between temperature and $E$ mode polarization as well as $C_{\ell}^{E E}$ and $C_{\ell}^{B B}$ which are the spectra of the $E$ and $B$ modes of polarization. The $T E$ and $E E$ spectra at large scales are used to estimate the reionization optical depth to high precision, a parameter to which the temperature power spectrum is not very sensitive. These spectra also give independent measurements of the other cosmological parameters estimated from the temperature power spectrum. This does not only serve as a consistency check, but also improves the statistical error bars on these parameters. Finally the much weaker $B B$ polarization power spectrum may at large scales be dominated by a signal arising from a background of gravitational waves which originated from inflation. This would be an important confirmation of the theory of inflation, and would as well give information about the detailed physics of the inflationary epoch. At smaller scales, the $B B$ spectrum is 
dominated by $E E$ modes which have been converted to $B B$ modes by gravitational lensing of large scale structure in the more recent universe.

Taking into account the huge amount of polarization data which will be available in the next 1-2 decades, as well as the important cosmological information contained in these data, it is clear that efficient data analysis tools will be necessary. While a large amount of data analysis techniques have been developed for analyzing CMB temperature data, much less attention has been given to analysis techniques for polarization data, also due to the lack of available high sensitivity observations.

An important tool for the analysis of temperature data has been various kinds of spherical wavelets (see [3, 4, 5] and the references therein). Indeed, in the last decade, wavelet methods have found applications in virtually all areas where statistical methods for CMB data analysis are required. Just to mention a few examples, we recall foreground subtraction ([6]), point source detection ([7, 8]), testing for non-Gaussianity (see [9, 10, 11]), search for anisotropies [12, 13], component separation ([14]), cross-correlation between CMB and Large Scale Structure Data ([15]), and many others. Directional wavelets have been advocated by [16, 17]. The rationale for such a widespread interest can be explained as follows: CMB models are best analyzed in the frequency domain, where the behaviour at different multipoles can be investigated separately; on the other hand, partial sky coverage and other missing observations make the evaluation of exact spherical harmonic transforms troublesome. The combination of these two features makes the time-frequency localization properties of wavelets most valuable.

More recently, a new kind of wavelets has found many fruitful applications in the CMB literature, the so-called spherical needlets. Spherical needlets were introduced in the functional analysis literature by [18, 19], and then considered for the statistical analysis of spherical random fields by $([20,21])$, with a view to applications to the statistical analysis of CMB data, including estimation of the angular power spectrum, testing for Gaussianity and bootstrap procedures. The related literature is already quite rich: applications to the analysis of the integrated Sachs-Wolfe effect by means of cross-correlation between CMB and Large Scale Structure data were given in [22]; a general introduction to their use for CMB data analysis is given in [23]; a discussion on optimal weight functions is provided in 24]; further applications include ([25], [26, 27, 28]). The extension to the construction of [18, 19] to the case of non-compactly supported weight functions is provided by [29, 30, 31], 
where the relationship with Spherical Mexican Hat Wavelets is also investigated, leading to the analysis of so-called Mexican Needlets. The stochastic properties of the corresponding Mexican needlet coefficients are established in [32, 33].

In this paper, we show how the scalar needlets which were applied to CMB temperature data can be extended to polarization using spin needlets. The latter were introduced in the

mathematical literature by [34]; they can be viewed as spin-2 wavelets designed for spin-2 fields on the sphere having the same properties when applied to CMB polarization fields as scalar needlets have when applied to CMB temperature fields. In particular, we shall show below that spin needlets enjoy both the localization and the uncorrelation properties that make scalar needlets a powerful tool for the analysis of CMB temperature data. The aim of this paper is then to introduce the spin-2 needlets, show how they can be applied to $Q$ and $U$ maps, and discuss some preliminary ideas for possible future statistical applications (how these maps can be reconstructed from the spin-2 needlet coefficients, how $E E$ and $B B$ spectra may be obtained directly from these coefficients, tests of non-Gaussianity and others).

The plan of the paper is as follows: in Section 2 we provide a quick review of standard (scalar) needlets and their main properties; in Section 3 we report the main results of [34], where spin needlets were first introduced and investigated from the mathematical point of view. In Section 4 we discuss a number of possible future applications to polarization data analysis; in Section 5 we provide a comparison with alternative approaches for the wavelet analysis of polarization data, while in Section 6 we present some preliminary evidence on the reconstruction properties of spin needlets. Some background material on spin spherical harmonics is given in the Appendix.

\section{A REVIEW OF SCALAR NEEDLETS}

To ease comparisons with the spin needlets which we will describe later, we recall very briefly the construction of a needlet basis. The spherical needlet (function) is defined as

$$
\psi_{j k}(\hat{\gamma})=\sqrt{\lambda_{j k}} \sum_{\ell} b\left(\frac{\ell}{B^{j}}\right) \sum_{m=-\ell}^{\ell} \bar{Y}_{\ell m}(\hat{\gamma}) Y_{\ell m}\left(\xi_{j k}\right)
$$

here, $\hat{\gamma}$ is a direction $(\theta, \phi)$ on the sphere, and $j$ is the frequency (multipole range) of the needlet. We use $\left\{\xi_{j k}\right\}$ to denote a set of cubature points on the sphere, corresponding to 
a given frequency $j$. In practice, we will identify these points with the pixel centres in the HEALPix pixelization scheme [38]. The cubature weights $\lambda_{j k}$ are inversely proportional to the number of pixels we are actually considering (see [22], [21] for more details); we can take for simplicity $\lambda_{j k}=4 \pi / N_{j}$, where here and throughout the paper we shall take $N_{j}$ to be the number of cubature points $\left\{\xi_{j k}\right\}$. Needlets can then be viewed as a combination, analogous to convolution, of the projection operators $\sum_{m=-\ell}^{\ell} \bar{Y}_{\ell m}(\hat{\gamma}) Y_{\ell m}\left(\xi_{j k}\right)$ with a suitably chosen window function $b(x)$ with $x=\ell / B^{j}$. The number $B$ appearing in the argument of the function $b(x)$ is a parameter which defines the needlet basis, as will be discussed below. Special properties of $b(x)$ ensure that the needlets enjoy quasi-exponential localization properties in pixel space. Formally, we must ensure that $([18,19])$ :

- A1 The function $b^{2}(x)$ is positive in the range $x=\left[\frac{1}{B}, B\right]$, zero otherwise; hence $b\left(\frac{\ell}{B^{j}}\right)$ is positive in $\ell \in\left[B^{j-1}, B^{j+1}\right]$

- A2 the function $b(x)$ is infinitely differentiable in $(0, \infty)$.

- A3 we have

$$
\sum_{j=1}^{\infty} b^{2}\left(\frac{\ell}{B^{j}}\right) \equiv 1 \text { for all } \ell>B .
$$

Condition A1 can be generalized to cover functions such as $x \exp (-x)$, thus leading to socalled Mexican needlets, see [29, 30, 31] where advantages and disadvantages of such choice are also discussed. In the present formulation, A1 ensures the needlets have bounded support in the harmonic domain; A2 is needed for the derivation of the localization properties, which we shall illustrate in the following section. Finally, A3 (the partition of unity property) is needed to establish the reconstruction formula (6) . Examples of constructions satisfying A1-A3 are given in [22, 24].

We shall now recall briefly some of the general features of needlets, which are not in general granted by other spherical wavelet constructions. We refer for instance to [23] for more details and a general introduction for a CMB readership. Briefly, we recall the following features:

a) needlets do not rely on any tangent plane approximation (compare [8]), and take advantage of the manifold structure of the sphere;

b) being defined in harmonic space, they are computationally very convenient, and inherently adapted to standard packages such as HEALPix; 
c) they allow for a simple reconstruction formula (see (땀), where the same needlet functions appear both in the direct and the inverse transform. This property is the same as for spherical harmonics but it is not shared by other wavelets systems;

d) they are quasi-exponentially (i.e. faster than any polynomial) concentrated in pixel space, see (71) below;

e) they are exactly localized on a finite number of multipoles; the width of this support is explicitly known and can be specified as an input parameter (see (1));

f) random needlet coefficients can be shown to be asymptotically uncorrelated (and hence, in the Gaussian case, independent) at any fixed angular distance, when the frequency increases (see [20]). This capital property can be exploited in several statistical procedures, as it allows one to treat needlet coefficients as a sample of independent and identically distributed coefficients on small scales, at least under the Gaussianity assumption.

More precisely, random needlet coefficients are given by

$$
\begin{aligned}
\beta_{j k} & =\int_{S^{2}} T(\hat{\gamma}) \psi_{j k}(\hat{\gamma}) d \hat{\gamma} \\
& =\sqrt{\lambda_{j k}} \sum_{\ell} b\left(\frac{\ell}{B^{j}}\right) \sum_{m=-\ell}^{\ell}\left\{\int_{S^{2}} T(\hat{\gamma}) \bar{Y}_{\ell m}(\hat{\gamma}) d \hat{\gamma}\right\} Y_{\ell m}\left(\xi_{j k}\right) \\
& =\sqrt{\lambda_{j k}} \sum_{\ell} b\left(\frac{\ell}{B^{j}}\right) \sum_{m=-\ell}^{\ell} a_{\ell m} Y_{\ell m}\left(\xi_{j k}\right) .
\end{aligned}
$$

Here $j$ denotes the frequency of the coefficient and $k$ refers to the direction $(\theta, \phi)$ on the sky. The index $k$ can in practice be the pixel number on the HEALPix grid. It is very important to stress that, although the needlets do not make up an orthonormal basis for square integrable functions on the sphere, they do represent a tight frame. In general, a tight frame on the sphere is a countable set of functions which preserves the norm; frames do not in general make up a basis, as they admit redundant elements. They can be viewed as the closest system to a basis, for a given redundancy, see [20, 31, 35] for further definitions and discussion. In our framework, the norm-preserving property becomes

$$
\sum_{j k} \beta_{j k}^{2} \equiv \int_{S^{2}} T^{2}(\hat{\gamma}) d \hat{\gamma}=\sum_{\ell=1}^{\infty}(2 \ell+1) \widehat{C}_{\ell}
$$

where

$$
\widehat{C}_{\ell}=\frac{1}{2 \ell+1} \sum_{m}\left|a_{\ell m}\right|^{2}
$$


is the raw angular power spectrum of the map $T(\hat{\gamma})$. (5) suggests immediately some procedures for angular power spectrum estimations and testing $([20,28])$ and is related to a much more fundamental result, i.e. the reconstruction formula

$$
T(\hat{\gamma}) \equiv \sum_{j, k} \beta_{j k} \psi_{j k}(\hat{\gamma})
$$

which in turn is a non-trivial consequence of the careful construction leading to (2). Again, we stress that the simple reconstruction formula of (66) is typical of tight frames but does not hold in general for other wavelet systems. It is easy to envisage many possible applications of (6) when handling masked data.

\section{A. Localization and uncorrelation properties}

The following quasi-exponential localization property of needlets is due to [18, 19] and motivates their name:

For any $M=1,2, \ldots$ there exists a positive constant $c_{M}$ such that for any point $\hat{\gamma} \in S^{2}$ we have

$$
\left|\psi_{j k}(\hat{\gamma})\right| \leq \frac{c_{M} B^{j}}{\left(1+B^{j} \arccos \left(\left|\hat{\gamma}-\xi_{j k}\right|\right)\right)^{M}} .
$$

We recall that $\arccos \left(\left|\hat{\gamma}-\xi_{j k}\right|\right)$ is just the geodesic distance on the unit sphere between the position $\hat{\gamma}$ and the position $\xi_{j k}$; (we recall in practice $\xi_{j k}$ can be the pixel center of a HEALPix pixel $k$ ). (7) is then stating that, for any fixed nonzero geodesic distance, the value of $\psi_{j k}(\hat{\gamma})$ goes to zero faster than any polynomial (quasi-exponentially) in the parameter $B$. Thus needlets achieve excellent localization properties in both the real and the harmonic domain. In [29], (77) is extended to the case of a non-compactly supported but smooth $b(x)$, thus covering also the Mexican needlet case (where $b(x) \simeq x \exp (-x)$ ).

From the stochastic point of view, the crucial uncorrelation properties for random spherical needlet coefficients were given in [20]. More precisely, two forms of uncorrelation were established

- P1 Whenever $\left|j_{1}-j_{2}\right| \geq 2$, we have that $\left\langle\beta_{j_{1} k_{1}} \beta_{j_{2} k_{2}}\right\rangle=0$, \langle\rangle denoting the expected value

- P2 For $j_{1}=j_{2}$, for any $M>0$ there exist a constant $C_{M}$ such that

$$
\frac{\left|\left\langle\beta_{j k} \beta_{j k^{\prime}}\right\rangle\right|}{\left\langle\beta_{j k}^{2}\right\rangle} \leq \frac{C_{M}}{\left(1+B^{j} \arccos \left(\left|\xi_{j k}-\xi_{j k^{\prime}}\right|\right)\right)^{M}} .
$$


Property P1 is a straightforward consequence of the localization properties for $b(x)$ in the harmonic domain. Property P2 is much more surprising, and does not follow by any means from the localization in pixel space (7); indeed it is simple to provide examples of wavelet systems that satisfy (77), and still do not enjoy (8)) (see [32], [33]). Both these properties hold for any isotropic random field, without any assumption on its distribution (i.e. Gaussianity).

Properties P1,P2 suggest that at high frequency, needlet coefficients can be approximated as a sample of identically distributed and uncorrelated (independent, in the Gaussian case) coefficients, and this property opens the way to a huge toolbox of statistical procedures for $\mathrm{CMB}$ data analysis. In practice, numerical approximations and the presence of masked regions will entail that P1 and P2 will only hold approximately; nevertheless, simulations have suggested that these properties do ensure a remarkable performance of needlets when applied to actual data from CMB experiments, see [29, 30, 31, 36] for further developments.

\section{SPIN NEEDLETS}

Throughout this paper, we shall assume that there exist a grid of cubature points $\left\{\xi_{j k}\right\}$, and a set of corresponding weights $\left\{\lambda_{j k}\right\}$ such that following discrete approximations of spherical integrals hold:

$$
\begin{aligned}
\sum_{k} \lambda_{j k}\left\{{ }_{2} Y_{\ell m}\left(\xi_{j k}\right)\right\} \overline{\left\{{ }_{2} Y_{\ell^{\prime} m^{\prime}}\left(\xi_{j k}\right)\right\}} & \left.\simeq \int_{S^{2}}{ }_{2} Y_{\ell m}(\hat{\gamma})\right\} \overline{\left.{ }_{2} Y_{\ell^{\prime} m^{\prime}}(\hat{\gamma})\right\}} d \hat{\gamma} \\
& =\delta_{l}^{l^{\prime}} \delta_{m}^{m^{\prime}} .
\end{aligned}
$$

Here ${ }_{ \pm 2} Y_{\ell m}$ are the spin spherical harmonics defined in the appendix. For the standard scalar case, the existence of such points is well-known and provided by many different constructions, see for instance $([18,19])$ and $([37])$, see also [20, 21] for further discussion and [29, 30, 31] for extensions to the generalized needlets case. For the spin spherical harmonics we consider here, the validity of (9) is going to be investigated mathematically elsewhere. We stress, however, that even if equation (9) is not known, or the points $\left\{\xi_{j k}\right\}$ are not explicitly given, then one can use any sensible collection of points and weights, and the results will still hold approximately; in other words, our results below will continue to hold with minor numerical approximations when implemented on any package with a pixelization scheme such as HEALPix (this issue is discussed rigorously and in greater detail in [34]). 
Spin needlets are defined as (see ([34] ) for a complete mathematical treatment and more rigorous results)

$$
\psi_{j k ; 2}(\hat{\gamma})=\sqrt{\lambda_{j k}} \sum_{\ell} b\left(\frac{\ell}{B^{j}}\right) \sum_{m=-\ell}^{\ell}\left\{{ }_{2} Y_{\ell m}(\hat{\gamma})\right\}\left\{\overline{{ }_{2} Y_{\ell m}\left(\xi_{j k}\right)}\right\} .
$$

A comparison between (1) and (10) highlights immediately that spin needlets make up a natural extension of the ideas underlying the approach in the scalar case to the framework of a spin field. This deep link between the two constructions should not hide, however, some profound differences between $\psi_{j k}(\hat{\gamma})$ and $\psi_{j k ; 2}(\hat{\gamma})$ as mathematical objects. Indeed, as recalled in the previous Section $\psi_{j k}(\hat{\gamma})$ is a standard scalar function which induces a linear map (3) leading from $T(\hat{\gamma}) \rightarrow \beta_{j k}$, i.e. from a scalar quantity to a scalar quantity. On the contrary, $\psi_{j k ; 2}$ induces a linear map leading from spin 2 quantities to spin 2 wavelet coefficients. The quantities in (10) depend on the choice of the coordinate system for $\hat{\gamma}$ and $\xi_{j k}$; these two coordinate systems may be chosen independently. If the coordinate system for $\hat{\gamma}$ is rotated, $\psi_{j k ; 2}(\hat{\gamma})$ transforms like a spin 2 vector at $\hat{\gamma}$, while if the coordinate system at $\xi_{j k}$ is rotated, $\psi_{j k ; 2}(\hat{\gamma})$ transforms like a spin -2 vector at $\xi_{j k} . \psi_{j k ; 2}(\hat{\gamma})$ has a precise mathematical status $($ see $([34]))$ as a linear map from spin 2 vectors at $\xi_{j k}$ to spin two vectors at $\hat{\gamma}$. Indeed, if $v$ is a spin 2 vector at $\xi_{j k}, \psi_{j k ; 2}(\hat{\gamma}) v$ makes sense as a spin 2 vector at $\hat{\gamma}$, since the product of a spin -2 vector and a spin 2 vector at a point is a well-defined complex number, independent of choice of coordinates. (Thus it would be more proper to write $\left\{{ }_{2} Y_{\ell m}(\hat{\gamma})\right\} \otimes\left\{\overline{{ }_{2} Y_{\ell m}\left(\xi_{j k}\right)}\right\}$ than $\left\{{ }_{2} Y_{\ell m}(\hat{\gamma})\right\}\left\{\overline{{ }_{2} Y_{\ell m}\left(\xi_{j k}\right)}\right\}$ in (10).)

As we shall show below the fact that $\psi_{j k ; 2}(\hat{\gamma})$ is not a scalar function does not prevent useful applications for the reconstruction and testing on physically meaningful scalar quantities such as the angular power spectra $C_{l}^{E E}, C_{l}^{B B}$. We note that in the mathematical results of [34], the factor $b\left(l / B^{j}\right)$ is replaced by $b\left(\sqrt{(l-2)(l+3)} / B^{j}\right)$; this reflects the fact that the sequence $\{(l-2)(l+3)\}_{l=3,4, \ldots}$ represents the eigenvalues of the Laplacian operator associated to spin spherical harmonics. However, since our main interest here is high-frequency asymptotics, and since $l \sim \sqrt{(l-2)(l+3)}$ for large $l$, we use the simpler $b\left(l / B^{j}\right)$ here to highlight the similarity with the usual presentation of scalar needlets. (Antithetically, one may modify the definition of the latter by replacing $b\left(l / B^{j}\right)$ with $b\left(\sqrt{l(l+1)} / B^{j}\right)$; note indeed that $\{l(l+1)\}_{l=1,2}$ provides the sequence of eigenvalues for the usual Laplacian operator on the sphere. We refer to [29, 30, 31] for more discussion on this point.)

In figure 1 we show the projection of $\psi_{j k ; 2}(\hat{\gamma})$ on the plane for $B=1.2$ and $j=10$. 

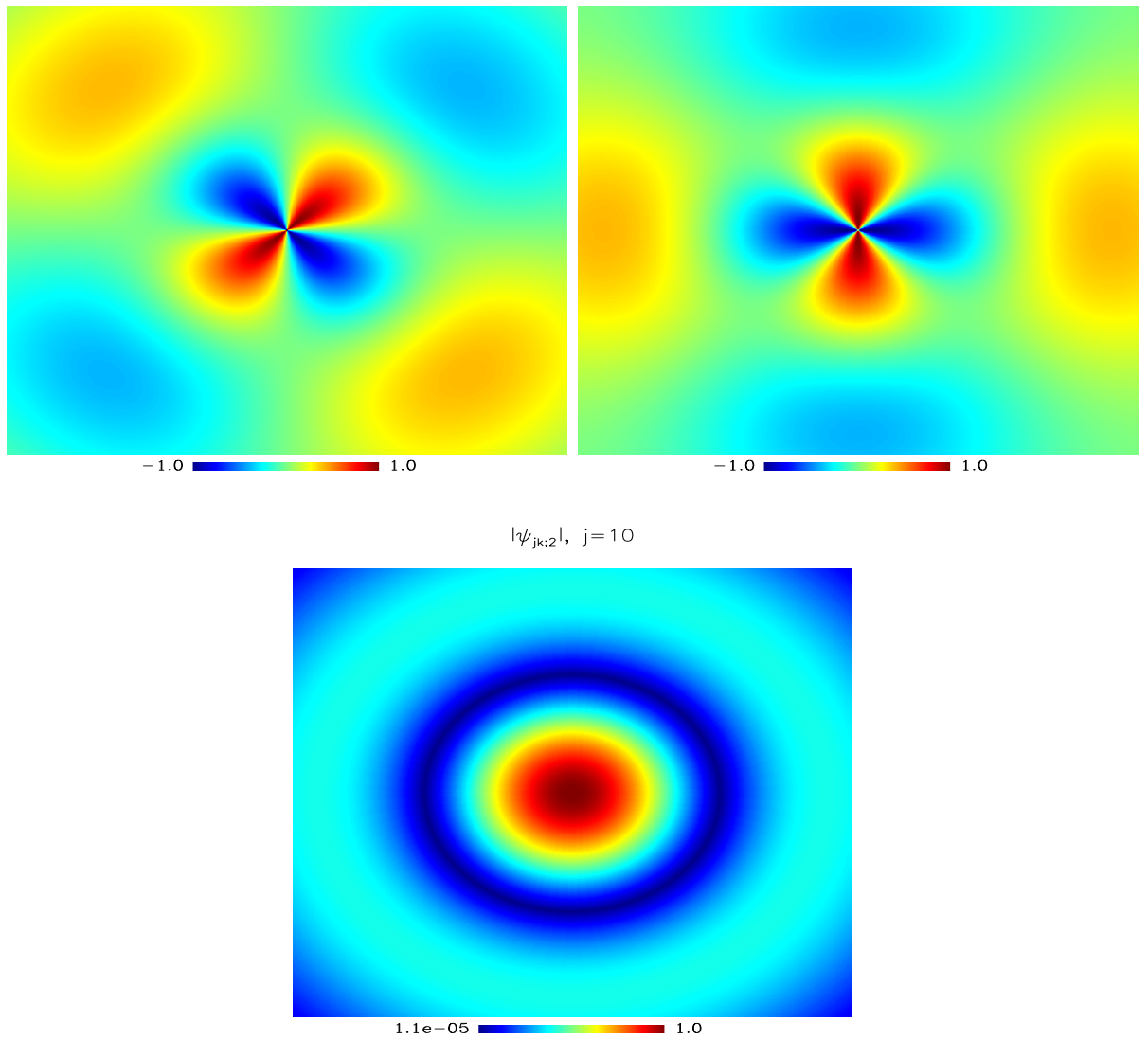

FIG. 1: Projections of $\psi_{j k ; 2}(\hat{\gamma})$ for $B=1.2$ and $j=10$. Left plot: real part, right plot: imaginary part, lower plot: modulus.

We show the real and imaginary component as well as the modulus. In figure 2 we show how $\psi_{j k ; 2}(\hat{\gamma})$ falls off with the distance from the center for $B=1.2$ and $j=10,20,30$. As expected, we clearly see how $\psi_{j k ; 2}(\hat{\gamma})$ falls of faster for higher values of $j$ and thus picking up smaller scales.

We shall now investigate the localization properties of (10). Localization properties in multipole space are a straightforward consequence of the properties of the weight function $b(x)$; localization properties in pixel space are much less straightforward and require rather sophisticated mathematical arguments. The following inequality is established in [34] and generalizes (7):

Proposition 1 [34] For any $M=1,2, \ldots$ there exists a positive constant $c_{M}$ such that 

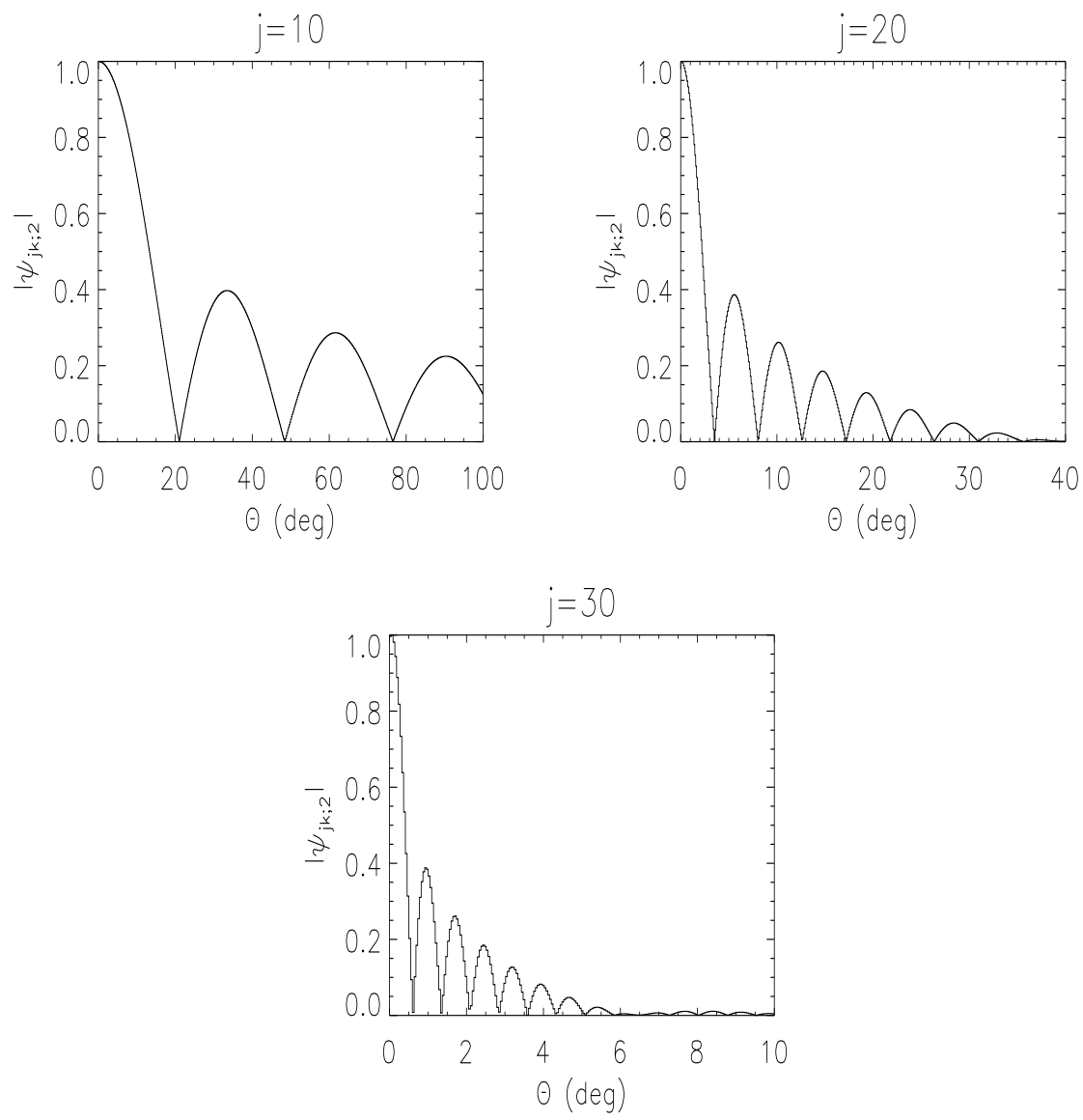

FIG. 2: The modulus of $\psi_{j k ; 2}(\hat{\gamma})$ for $B=1.2$ and $j=10$ (left plot), $j=20$ (right plot) and $j=30$ (lower plot). The angle $\theta$ is the distance from the center point.

for any point $\hat{\gamma} \in S^{2}$ we have

$$
\left|\psi_{j k ; 2}(\hat{\gamma})\right| \leq \frac{c_{M} B^{j}}{\left(1+B^{j} \arccos \left(\left|\hat{\gamma}-\xi_{j k}\right|\right)\right)^{M}}
$$

Note that although $\left\{\psi_{j k ; 2}(\hat{\gamma})\right\}$ are not scalar valued, $\left|\psi_{j k ; 2}(\hat{\gamma})\right|$ is clearly a well-defined scalar function, so that the inequalities (11) are consistent.

Now consider the spin 2 fields

$$
Q(\hat{\gamma})+i U(\hat{\gamma})=\sum_{l m} a_{l m ; 2}\left\{{ }_{2} Y_{l m}(\hat{\gamma})\right\}
$$

where we have introduced the complex-valued random coefficients

$$
a_{l m ; 2}=\int_{S^{2}}\{Q(\hat{\gamma})+i U(\hat{\gamma})\} \overline{\left\{{ }_{2} Y_{l m}(\hat{\gamma})\right\}} d \hat{\gamma}=-\left(a_{l m ; E}+i a_{l m ; B}\right)
$$


where $a_{l m ; E}, a_{l m ; B}$ denote, respectively, the spherical harmonics coefficients of the $E, B$ components of the polarization random field. The spin needlet coefficients are defined as

$$
\begin{aligned}
\beta_{j k ; 2} & :=\int_{S^{2}}\{Q(\hat{\gamma})+i U(\hat{\gamma})\} \overline{\psi_{j k ; 2}(\hat{\gamma})} d \hat{\gamma} \\
& =\sqrt{\lambda_{j k}} \sum_{l m} b\left(\frac{l}{B^{j}}\right) a_{l m ; 2}\left\{{ }_{2} Y_{l m}\left(\xi_{j k}\right)\right\},
\end{aligned}
$$

in obvious analogy to (44) for the scalar case, only replacing spin spherical harmonics and the corresponding (scalar) random coefficients. Note that

$$
\overline{\psi_{j k ; 2}(\hat{\gamma})}=\sqrt{\lambda_{j k}} \sum_{\ell} b\left(\frac{\ell}{B^{j}}\right) \sum_{m=-\ell}^{\ell}\left\{\overline{{ }_{2} Y_{\ell m}(\hat{\gamma})}\right\}\left\{{ }_{2} Y_{\ell m}\left(\xi_{j k}\right)\right\}
$$

is a spin 2 vector at $\xi_{j k}$ and a spin -2 vector at $\hat{\gamma} ; \beta_{j k ; 2}$ is a spin 2 vector at $\xi_{j k}$. In other words, $Q(\hat{\gamma})+i U(\hat{\gamma})=\sum_{l m} a_{l m ; 2}\left\{2 Y_{l m}(\hat{\gamma})\right\}$ is spin 2 ; when multiplied with $\overline{\psi_{j k ; 2}(\hat{\gamma})}$, the spin 2 factor annihilates with the spin -2 factor in $\overline{{ }_{2} Y_{\ell m}(\hat{\gamma})}$, and we are just left with a spin 2 factor in $\beta_{j k ; 2}$, given by $\left\{{ }_{2} Y_{l m}\left(\xi_{j k}\right)\right\}$. In figure 3 and 4 we show maps of the amplitude and direction of polarization of the input map as well as for needlet coefficients at $j=10,20,30$ for $B=1.2$.

Assuming (9), the following reconstruction formulae can be shown to hold (see ([34])

$$
\{Q(\hat{\gamma})+i U(\hat{\gamma})\}=\sum_{j k} \beta_{j k ; 2} \psi_{j k ; 2}(\hat{\gamma}) .
$$

Note that the right side of (13) makes sense, independent of coordinate system chosen for the $\xi_{j k}$, and defines a spin 2 vector at $\hat{\gamma}$. To see this, one need only note again that the product of two quantities, one which transforms like a spin 2 vector at $\xi_{j k}$, and the other which transforms like a spin -2 vector at $\xi_{j k}$, is a well-defined complex number, independent of choice of coordinate system. An example of the reconstruction is shown in figure 5 . In the figure we show the relative difference in percent between the input and reconstructed $\mathrm{Q}$ and $\mathrm{U}$ maps. Outside the masked regions, the difference is equal to the numerical noise obtained by doing a simple spherical harmonic transform followed by the inverse transform. Close to the masked regions, there is a small area where the reconstruction error is larger. The size of this region will depend on the choice of $B$. In this case $B=1.2$ which is a rather low value resulting in a relatively large region with larger error around the mask. Note that most of these points still have reconstruction errors smaller than $5 \%$. We will discuss the accuracy of reconstruction in detail in a following paper. 

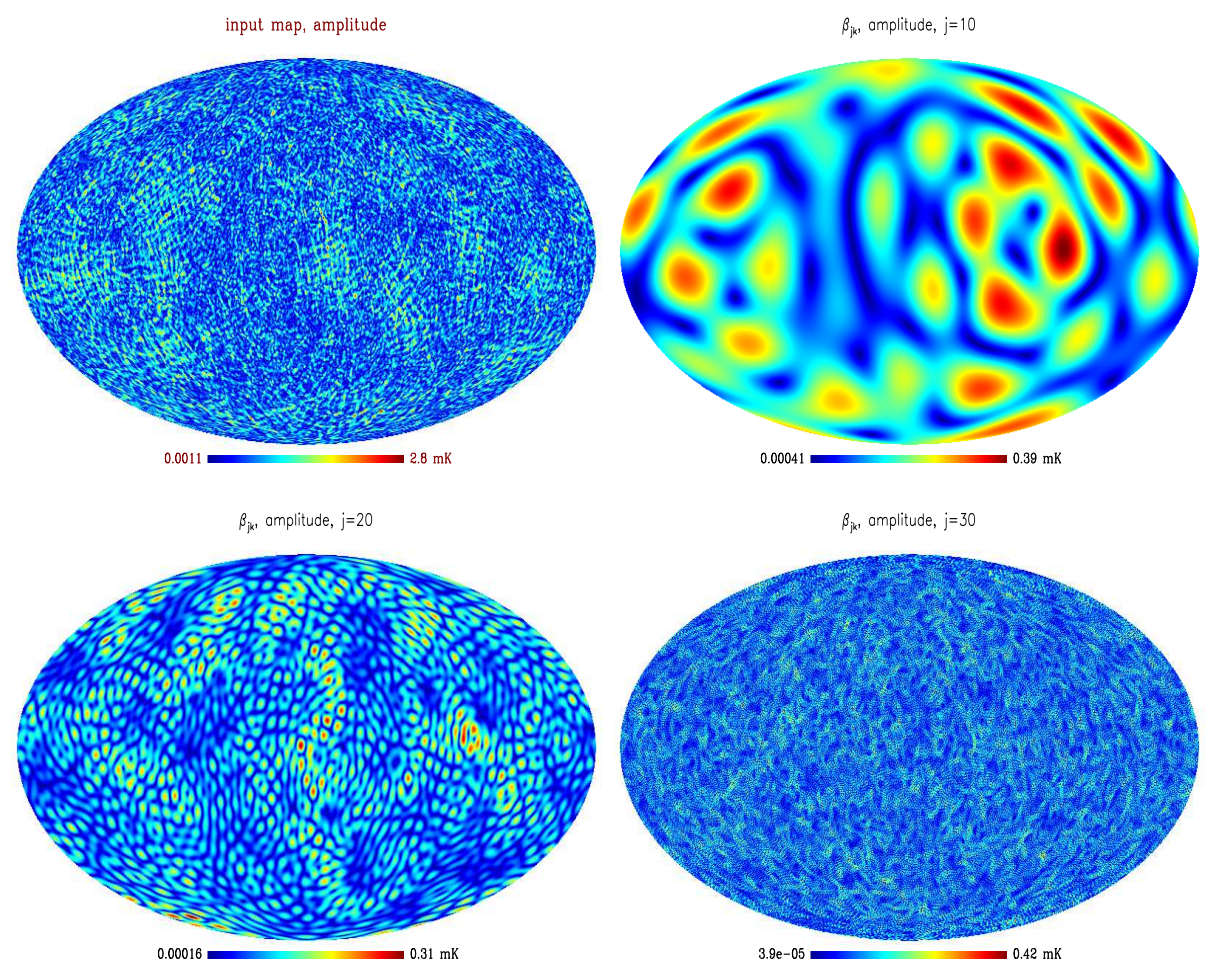

FIG. 3: The input CMB map as well as the needlets coefficients for $B=1.2$ and $j=10,20$ and 30 . The plots show the polarization amplitude.

As detailed in the previous Section, a capital property for the random needlet coefficients in the scalar case is asymptotic uncorrelation at any fixed angular distance (for a smooth angular power spectrum), as the frequency parameter diverges $(j \rightarrow \infty)$. A natural question is the extent to which this property may continue to hold in the spin case. The answer to this question is provided in [34], where it is shown that, under mild regularity conditions on the angular power spectra, for all $M>0$ there exist positive constants $C_{M}$ such that

$$
\frac{\left|\left\langle\beta_{j k ; 2} \beta_{j k^{\prime} ; 2}\right\rangle\right|}{\left\langle\left|\beta_{j k ; 2}\right|^{2}\right\rangle} \leq \frac{C_{M}}{\left(1+B^{j} \arccos \left(\left|\xi_{j k}-\xi_{j k^{\prime}}\right|\right)\right)^{M}} .
$$

Again, $\left|\left\langle\beta_{j k ; 2} \beta_{j k^{\prime} ; 2}\right\rangle\right|$ is a well defined scalar functions, despite the fact that $\left\langle\beta_{j k ; 2} \beta_{j k^{\prime} ; 2}\right\rangle$ are spin quantities that depend on the choice of coordinates for tangent planes. (14) suggests that the spin spherical needlet coefficients can be consistently used for angular power spectrum estimation and map reconstruction, as detailed in the following section; in the scalar case, this argument is rigorously derived in $([20])$, whereas for the spin situation we are concerned with here, we refer again to [34] for a more complete mathematical analysis. 

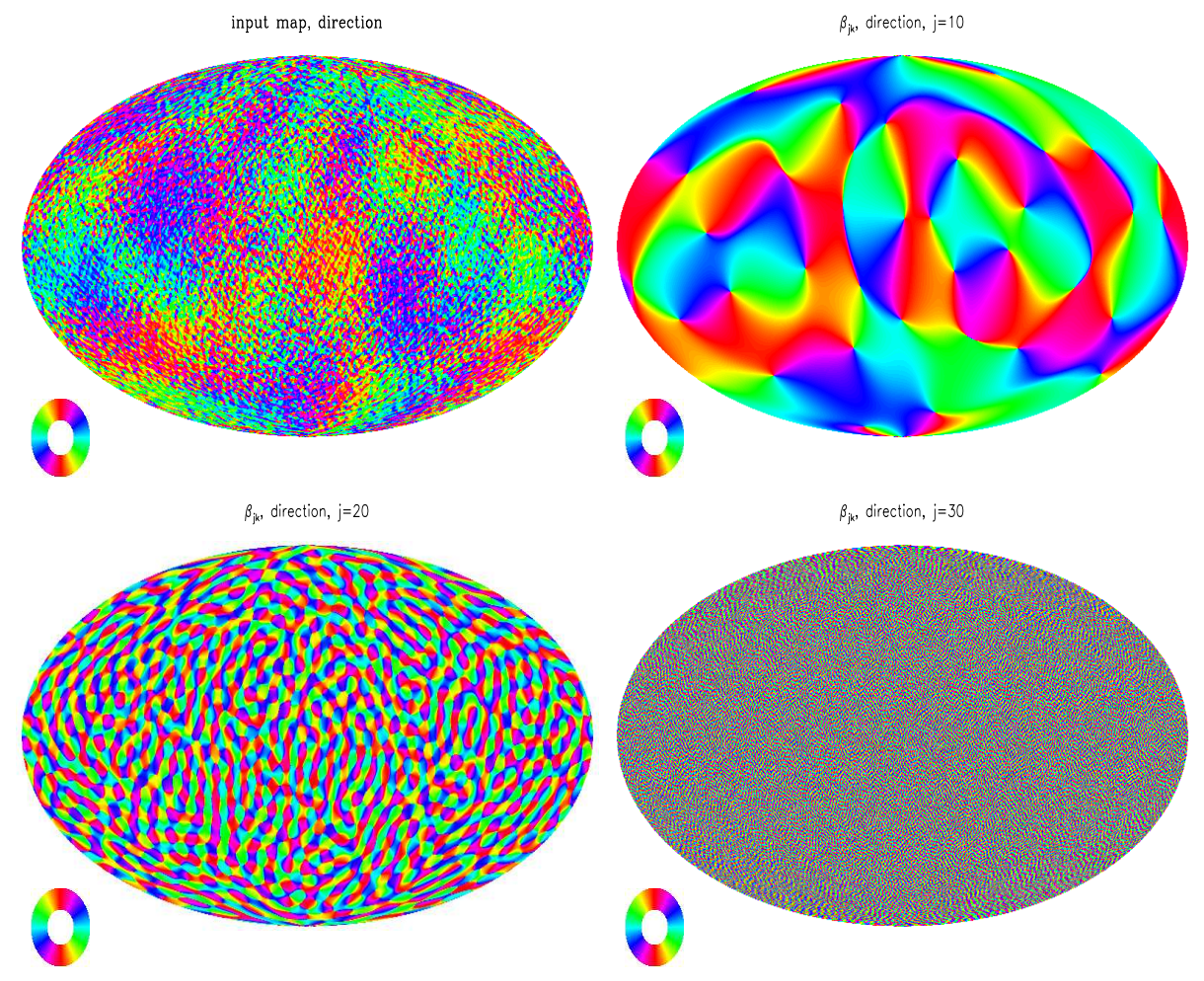

FIG. 4: The input CMB map as well as the needlets coefficients for $B=1.2$ and $j=10,20$ and 30 . The plots show the polarization direction.
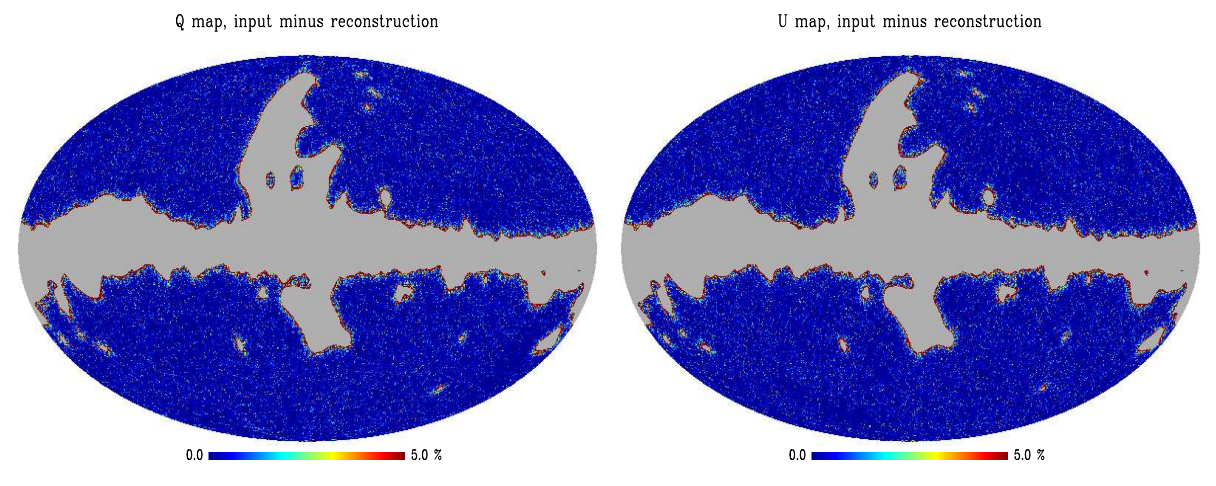

FIG. 5: The relative difference (in \% between an input CMB map ( $\mathrm{Q}$ and $\mathrm{U}$ map) and the reconstructed map in the presence of a mask (in this case the P06 galactic cut used by the WMAP team for polarisation). 
Now assume that we have a masked region $M$ where no polarization data are actually available; of course, this implies that we shall only be able to recover the coefficients

$$
\widetilde{a}_{l m ; 2}=\int_{S^{2} / M}\{Q(\hat{\gamma})+i U(\hat{\gamma})\} \overline{\left\{{ }_{2} Y_{l m}(\hat{\gamma})\right\}} d \hat{\gamma} .
$$

Due to the poor localization properties of spherical harmonics, the coefficients $\left\{\widetilde{a}_{l m ; 2}\right\}$ need not be close in any meaningful sense to $\left\{a_{l m ; 2}\right\}$; consequently any statistical procedure based naively upon them (including estimation of $C_{l}^{E E}, C_{l}^{B B}$ or the reconstruction of the scalar maps $E(\widehat{\gamma}), B(\widehat{\gamma}))$ is likely to be seriously flawed. On the other hand, consider

$$
\widetilde{\beta}_{j k ; 2}=\sqrt{\lambda_{j k}} \sum_{l m} b\left(\frac{l}{B^{j}}\right) \widetilde{a}_{l m ; 2}\left\{{ }_{2} Y_{l m}\left(\xi_{j k}\right)\right\} ;
$$

for locations $\left\{\xi_{j k}\right\}$ that are "sufficiently away" from the masked region $M$, i.e. $d\left(\xi_{j k}, M\right)>$ $\delta>0$, it is easy to see that (see (11))

$$
\begin{aligned}
\left\langle\left|\widetilde{\beta}_{j k ; 2}-\beta_{j k ; 2}\right|\right\rangle & =\left\langle\left|\int_{M}\{Q(\hat{\gamma})+i U(\hat{\gamma})\} \overline{\psi_{j k ; 2}(\hat{\gamma})} d \hat{\gamma}\right|\right\rangle \\
& \leq \frac{c_{M} B^{j}}{\left(1+B^{j} \arccos (\delta)\right)^{M}}\left\langle\int_{M}|\{Q(\hat{\gamma})+i U(\hat{\gamma})\}| d \hat{\gamma}\right\rangle \\
& =o\left(B^{-j(M-1)}\right),
\end{aligned}
$$

i.e. the coefficients $\widetilde{\beta}_{j k ; 2}, \beta_{j k ; 2}$ become asymptotically equivalent at high frequencies $j \rightarrow \infty$.

It should be noted that throughout this paper we have decided to adopt the spin spherical harmonics formalism considered by [39]. A completely analogous result could have been obtained by taking combinations of $b\left(l / B^{j}\right)$ with grad and curl harmonics, i.e. starting from the approach of [40]. We do not focus on this possibility here for brevity's sake.

\section{SOME STATISTICAL APPLICATIONS}

The purpose of this Section is to provide some examples of the statistical applications which can be entertained by means of spin needlet coefficients. Our purpose here is not to provide recipes which are ready-to-use for CMB data analysis - this certainly requires much more computational and analytical work to take into account the features of CMB maps: foregrounds, anisotropic noise, multichannel observations and many others. Our purpose here is different: it is well-known how wavelets in the scalar case have proved to be a valuable instrument when dealing with a variety of data analysis issues. The discussion 
below suggests that spin needlets can be just as important. Due to their specific nature as mathematical (spin) objects, these applications however require extra care, even in the idealistic circumstances we focus on here.

\section{A. Estimation of the sum of the angular power spectra $C_{l}^{E E}+C_{l}^{B B}$}

We recall that an estimator of the binned temperature power spectrum can be constructed from scalar needlets, as follows. Take

$$
\widehat{\Gamma}_{j} \stackrel{\text { def }}{=} \sum_{k} \beta_{j k}^{2}
$$

in the idealistic case of no mask, it is readily seen that

$$
\left\langle\widehat{\Gamma}_{j}\right\rangle=\sum_{k}\left\langle\beta_{j k}^{2}\right\rangle=\sum_{B^{j-1}<l<B^{j+1}} b^{2}\left(\frac{l}{B^{j}}\right)(2 l+1) C_{l} \stackrel{\text { def }}{=} \Gamma_{j} .
$$

In the idealistic circumstances where the sky is fully-observed, the previous sum includes $N_{j}$ terms, where we recall that $N_{j}$ is the number of pixels at the resolution $j$. The result continues to hold (with some corrections to take into account the fraction of sky coverage) in the presence of a sky cut, although clearly $\sum_{k}$ runs over a smaller number of terms (in other words, a sky-fraction correction factor must be introduced). This estimator was proposed in $([20,22])$; in [22] applications are also shown to the cross-correlation between CMB and Large Scale Structure data, in [27, 28] this estimator is extended to allow for the presence of observational noise, whereas in [41] the same approach is applied to search for features and asymmetries in CMB maps. In $([20])$ it is also shown that, in the Gaussian case

$$
\frac{\widehat{\Gamma}_{j}-\Gamma_{j}}{\operatorname{Var}\left\{\widehat{\Gamma}_{j}\right\}} \rightarrow_{d} N(0,1)
$$

where $\rightarrow_{d}$ denotes convergence in distribution and $N(0,1)$ a standard Gaussian law. A result like (17) provides also a clue for testing goodness-of-fit and driving confidence intervals (at high frequencies) for the angular power spectra. In [21] the behaviour of statistics such as $\widehat{\Gamma}_{j}$ are considered for partial regions of the sky, also as a device for testing asymmetries. From the mathematical point of view, results like (17) are entirely justifiable on the basis of the uncorrelation properties we discussed earlier in P1-P2 (see (8) ). As the same sort of uncorrelation property has been established in (14), it is natural to investigate whether a similar procedure for the estimation of the binned angular power spectra is feasible here. 
The answer turns out to be positive, in the following sense. Consider the (scalar valued) estimator

$$
\widehat{\Gamma}_{j ; 2}=\sum_{k}\left|\beta_{j k ; 2}\right|^{2}
$$

Starting from the idealistic case of no sky-cuts, we obtain easily

$$
\begin{aligned}
\left\langle\widehat{\Gamma}_{j ; 2}\right\rangle & =\sum_{k}\left\langle\left|\beta_{j k ; 2}\right|^{2}\right\rangle \\
& =\sum_{k}\left\langle\left|\sqrt{\lambda_{j k}} \sum_{l m} b\left(\frac{l}{B^{j}}\right) a_{l m ; 2}\left\{{ }_{2} Y_{l m}\left(\xi_{j k}\right)\right\}\right|^{2}\right\rangle \\
& =\sum_{k} \lambda_{j k} \sum_{l} b^{2}\left(\frac{l}{B^{j}}\right) \Gamma_{l} \sum_{m}\left|\left\{{ }_{2} Y_{l m}\left(\xi_{j k}\right)\right\}\right|^{2} \\
& =\sum_{l} b^{2}\left(\frac{l}{B^{j}}\right) \Gamma_{l}(2 l+1) \stackrel{\text { def }}{=} \Gamma_{j ; 2},
\end{aligned}
$$

because

$$
\sum_{m}\left|\left\{{ }_{2} Y_{l m}\left(\xi_{j k}\right)\right\}\right|^{2}=\frac{2 l+1}{4 \pi},
$$

and as before we took $\lambda_{j k}=4 \pi / N_{j}$. Here

$$
\Gamma_{l}=<\left|a_{l m ; 2}\right|^{2}>=<\left|a_{l m E}+i a_{l m B}\right|^{2}>=C_{l}^{E E}+C_{l}^{B B}
$$

Likewise, it is possible to show that $([\underline{34}])$

$$
\frac{\widehat{\Gamma}_{j ; 2}-\Gamma_{j ; 2}}{\operatorname{Var}\left\{\widehat{\Gamma}_{j ; 2}\right\}} \rightarrow_{d} N(0,1), \text { as } j \rightarrow \infty,
$$

i.e. it is possible to prove that $\widehat{\Gamma}_{j ; 2}$ is consistent and asymptotically Gaussian around its expected value $\Gamma_{j ; 2}$. Note that, as the electric and magnetic components of the polarization field are uncorrelated, the variance in the denominator is simply the sum of the variances of the two scalar components. Likewise, the asymptotic theory can be developed as in [20, 21]. As mentioned before, the presence of masked regions of the sky requires the introduction of a sky coverage fraction. The presence of anisotropic noise is more interesting and can be dealt with along the lines of $([27,28])$, i.e. by introducing weighted, rather than simple, averages of the squared needlet coefficients.

The previous procedure allows one to estimate (a binned form of) the angular power spectra $C_{l}^{E E}+C_{l}^{B B}$. Although this could be sufficient for some purposes, it is clear that for $\mathrm{CMB}$ applications we are interested, in general, in the estimation of $C_{l}^{E E}$ and $C_{l}^{B B}$ as separate quantities; this is an issue to which we shall come back later in this Section. 


\section{B. Testing for non-Gaussianity}

As a further statistical application, it is possible to consider the investigation of nonGaussianity in the joint law of temperature and polarization data. A standard idea to focus on non-Gaussianity is to consider the skewness and kurtosis of wavelet coefficients; for brevity, we shall concentrate on the latter statistic. To normalize our coefficients, we estimate their variance by

$$
\widehat{\sigma}_{j}^{2} \stackrel{\text { def }}{=} \frac{1}{N_{j}} \sum_{k}\left|\beta_{j k ; 2}\right|^{2}=\frac{\widehat{\Gamma}_{j}}{N_{j}}
$$

where we define also

$$
\sigma_{j}^{2} \stackrel{\text { def }}{=}<\widehat{\sigma}_{j}^{2}>=\frac{1}{N_{j}} \sum_{k}<\left|\beta_{j k ; 2}\right|^{2}>.
$$

As a consequence of (18) and along the same lines as in [20], it can be shown that

$$
p \lim _{j \rightarrow \infty} \frac{\widehat{\sigma}_{j}^{2}}{\sigma_{j}^{2}}=p \lim _{j \rightarrow \infty} \frac{\widehat{\Gamma}_{j}}{\Gamma_{j}}=1,
$$

$p \lim _{j \rightarrow \infty}$ denoting as before convergence in probability; loosely speaking, this is to say that the mean of the squared spin needlet coefficients is a consistent estimator of their variance. We focus then on the normalized coefficients

$$
\widehat{\beta}_{j k ; 2} \stackrel{\operatorname{def}}{=} \frac{\beta_{j k ; 2}}{\widehat{\sigma}_{j}} ;
$$

to test for the joint Gaussianity of the temperature and polarization fields, we may consider for instance their kurtosis, i.e.

$$
\widehat{K}_{j ; P P}=\sum_{k}\left\{\left|\widehat{\beta}_{j k ; 2}\right|^{4}-3\right\},
$$

which converges to zero in probability as $j \rightarrow \infty$. More interestingly, we might be interested in testing for the joint Gaussianity of polarization and temperature maps. Likewise, it seems possible to focus on the needlet bispectrum for joint temperature and polarization data, as suggested for the scalar case by [25]. These issues will be developed in a future paper.

\section{Reconstruction of the $E$ and $B$ maps}

A common strategy to reconstruct the (scalar) maps of the electric and magnetic (or grad and curl) components of the polarization field is well-known; starting from the maps 
$\{Q \pm i U\}$, the scalar coefficients are evaluated by means of the Fourier inversions (12). The maps are then recovered by the standard spectral expansion. As simple as this procedure can seem, it is prone to severe problems in the analysis of actual data, where the presence of masked regions can lead to severe errors in the evaluation of exact Fourier transforms. We shall show here how one can use needlets to produce scalar maps by a novel technique.

We recall first how to obtain scalar maps from polarization data; indeed, as in ([42]) we focus on

$$
\widetilde{E}(\hat{\gamma}) \equiv-\frac{1}{2}\left[(\bar{\partial})^{2}(Q+i U)(\hat{\gamma})+(\partial)^{2}(Q-i U)(\hat{\gamma})\right]
$$

where we have used the spin raising and spin lowering operators $(\partial, \bar{\partial})$ defined in the appendix. The crucial property to recall is

$$
\begin{aligned}
& (\bar{\partial})^{2}\left\{{ }_{2} Y_{\ell m}(\widehat{\gamma})\right\}=\sqrt{\frac{(\ell+2) !}{(\ell-2) !}} Y_{\ell m}(\widehat{\gamma}), \\
& (\partial)^{2}\left\{\overline{{ }_{2} Y_{\ell m}}(\widehat{\gamma})\right\}=\sqrt{\frac{(\ell+2) !}{(\ell-2) !}} \overline{Y_{\ell m}}(\widehat{\gamma}) .
\end{aligned}
$$

Hence we obtain, in view of (13)

$$
\begin{aligned}
\widetilde{E}(\hat{\gamma})= & -\frac{1}{2}\left[(\bar{\partial})^{2} \sum_{j k} \beta_{j k ; 2} \psi_{j k ; 2}(\hat{\gamma})+(\partial)^{2} \sum_{j k} \overline{\beta_{j k ; 2} \psi_{j k ; 2}}(\hat{\gamma})\right] \\
= & -\frac{1}{2} \sum_{j k} \beta_{j k ; 2} \sum_{\ell m} b\left(\frac{\ell}{B^{j}}\right) \sqrt{\frac{(\ell+2) !}{(\ell-2) !}}\left\{Y_{\ell m}(\hat{\gamma})\right\}\left\{\overline{Y_{\ell m}}\left(\xi_{j k}\right)\right\} \\
& -\frac{1}{2} \sum_{j k} \overline{\beta_{j k ; 2}} \sum_{\ell m} b\left(\frac{\ell}{B^{j}}\right) \sqrt{\frac{(\ell+2) !}{(\ell-2) !}}\left\{\overline{Y_{\ell m}}(\hat{\gamma})\right\}\left\{{ }_{2} Y_{\ell m}\left(\xi_{j k}\right)\right\} \\
& =-\frac{1}{2} \sum_{j k}\left\{\beta_{j k ; 2} \varphi_{j k}(\hat{\gamma})+\overline{\beta_{j k ; 2}} \overline{\varphi_{j k}}(\hat{\gamma})\right\}
\end{aligned}
$$

where

$$
\begin{aligned}
& \varphi_{j k}(\hat{\gamma}) \stackrel{\text { def }}{=} \sqrt{\lambda_{j k}} \sum_{\ell m} b\left(\frac{\ell}{B^{j}}\right) \sqrt{\frac{(\ell+2) !}{(\ell-2) !}}\left\{Y_{\ell m}(\hat{\gamma})\right\}\left\{\overline{{ }_{2} Y_{\ell m}}\left(\xi_{j k}\right)\right\} \\
= & (\bar{\partial})^{2} \psi_{j k ; 2}(\hat{\gamma}),
\end{aligned}
$$


which is scalar in $\hat{\gamma}$, spin -2 in $\xi_{j k}$. Likewise

$$
\begin{aligned}
& \widetilde{B}(\hat{\gamma}) \equiv \frac{i}{2}\left[(\bar{\partial})^{2}(Q+i U)(\hat{\gamma})-(\partial)^{2}(Q-i U)(\hat{\gamma})\right] \\
& =\frac{i}{2}\left[(\bar{\partial})^{2} \sum_{j k} \beta_{j k ; 2} \psi_{j k ; 2}(\hat{\gamma})+(\partial)^{2} \sum_{j k} \overline{\beta_{j k ; 2} \psi_{j k ; 2}}(\hat{\gamma})\right] \\
& =\frac{i}{2} \sum_{j k} \beta_{j k ; 2} \sum_{\ell m} b\left(\frac{\ell}{B^{j}}\right) \sqrt{\frac{(\ell+2) !}{(\ell-2) !}}\left\{Y_{\ell m}(\hat{\gamma})\right\}\left\{\overline{{ }_{2} Y_{\ell m}}\left(\xi_{j k}\right)\right\} \\
& -\frac{i}{2} \sum_{j k} \overline{\beta_{j k ; 2}} \sum_{\ell m} b\left(\frac{\ell}{B^{j}}\right) \sqrt{\frac{(\ell+2) !}{(\ell-2) !}}\left\{\overline{Y_{\ell m}}(\hat{\gamma})\right\}\left\{{ }_{2} Y_{\ell m}\left(\xi_{j k}\right)\right\} \\
& =\frac{i}{2} \sum_{j k}\left\{\beta_{j k ; 2} \varphi_{j k}(\hat{\gamma})-\overline{\beta_{j k ; 2}} \overline{\varphi_{j k}}(\hat{\gamma})\right\} .
\end{aligned}
$$

In the presence of fully observed maps, it is immediate that

$$
\begin{gathered}
\widetilde{E}(\hat{\gamma})=-\frac{1}{2} \sum_{j k} \sqrt{\lambda_{j k}}\left\{\sum_{l m} \sqrt{\lambda_{j k}} b\left(\frac{l}{B^{j}}\right) a_{l m ; 2}\left({ }_{2} Y_{l m}\left(\xi_{j k}\right)\right)\right\} \sum_{\ell m^{\prime}} b\left(\frac{\ell}{B^{j}}\right) \sqrt{\frac{(\ell+2) !}{(\ell-2) !}}\left\{Y_{\ell m^{\prime}}(\hat{\gamma})\right\}\left\{\overline{{ }_{2} Y_{\ell m^{\prime}}}\left(\xi_{j k}\right)\right\} \\
-\frac{1}{2} \sum_{j k} \sqrt{\lambda_{j k}}\left\{\sum_{l m} \sqrt{\lambda_{j k}} b\left(\frac{l}{B^{j}}\right) \bar{a}_{l m ; 2}\left(\overline{{ }_{2} Y_{l m}}\left(\xi_{j k}\right)\right)\right\} \sum_{\ell m^{\prime}} b\left(\frac{\ell}{B^{j}}\right) \sqrt{\frac{(\ell+2) !}{(\ell-2) !}}\left\{\overline{Y_{\ell m^{\prime}}}(\hat{\gamma})\right\}\left\{{ }_{2} Y_{\ell m^{\prime}}\left(\xi_{j k}\right)\right\} \\
=-\frac{1}{2} \sum_{l m}\left(a_{l m ; 2}+\bar{a}_{l-m ; 2}\right) Y_{\ell m^{\prime}}(\hat{\gamma}) \sqrt{\frac{(\ell+2) !}{(\ell-2) !}} \sum_{j} b\left(\frac{l}{B^{j}}\right) b\left(\frac{\ell}{B^{j}}\right) \delta_{l}^{\ell} \delta_{m}^{m \prime} \\
=\sum_{l m} a_{l m ; E} \sqrt{\frac{(\ell+2) !}{(\ell-2) !}} Y_{l m}(\hat{\gamma})
\end{gathered}
$$

likewise, it is immediate that

$$
\widetilde{B}(\hat{\gamma})=\sum_{l m} a_{l m ; B} \sqrt{\frac{(l+2) !}{(l-2) !}} Y_{l m}(\hat{\gamma}),
$$

as expected. This is just a rephrasing of (13) in terms of the underlying (electric and magnetic) scalar fields.

In the presence of masked maps, $E$ and $B$ are unfeasible; our idea is then to use thresholding techniques, which have been shown to be powerful when combined with the wavelet approach (see for instance [43], or [28] for applications in a CMB framework). In particular, assume a portion $M$ of $S^{2}$ is masked; for each pixel $\left\{\xi_{j k}\right\}$ we can define the fraction

$$
s_{j k}=\frac{\int_{S^{2} / M}\left|\psi_{j k ; 2}(\hat{\gamma})\right|^{2} d \hat{\gamma}}{\int_{S^{2}}\left|\psi_{j k ; 2}(\hat{\gamma})\right|^{2} d \hat{\gamma}} .
$$


This ratio is clearly measuring the amount by which the needlet coefficient localized at $\left\{\xi_{j k}\right\}$ is corrupted by the presence of missing observations. Note that, due to the localization properties of spin needlets, $s_{j k}$ will converge to unity for all pixels $\left\{\xi_{j k}\right\}$ which are outside the masked regions. We can now define the thresholded parameters

$$
\beta_{j k ; 2}^{*}=\beta_{j k ; 2} \mathbb{I}\left(s_{j k}>t_{j}\right)
$$

where the function $\mathbb{I}\left(s_{j k}>t_{j}\right)$ takes the value one if $s_{j k}>t_{j}$, zero otherwise; $t_{j}$ is a thresholding parameter which is assumed to converge to one as $j \rightarrow \infty$. We can then consider the reconstructed maps

$$
\begin{gathered}
\widetilde{E}^{*}(\hat{\gamma}) \equiv-\frac{1}{2}\left\{\sum_{j k}\left[\beta_{j k ; 2}^{*} \varphi_{j k}(\hat{\gamma})+\overline{\beta_{j k ; 2}^{*}} \overline{\varphi_{j k}}(\hat{\gamma})\right]\right\}, \\
\widetilde{B}^{*}(\hat{\gamma}) \equiv \frac{i}{2} \sum_{j k}\left[\beta_{j k ; 2}^{*} \varphi_{j k}(\hat{\gamma})-\overline{\beta_{j k ;}^{*}} \overline{\varphi_{j k}}(\hat{\gamma})\right] .
\end{gathered}
$$

In view of (16), we expect $\widetilde{E}^{*}(\hat{\gamma}) \simeq \widetilde{E}(\hat{\gamma}), \widetilde{B}^{*}(\hat{\gamma}) \simeq \widetilde{B}(\hat{\gamma})$; the validity of these approximations depends upon the distance of $\hat{\gamma}$ from the masked region and the cosmic variance (i.e., the lower the value of $\left\{C_{l}^{E E}, C_{l}^{B B}\right\}$ at low multipoles, the better the approximation). The accuracy of this approach will be tested extensively in a future publication.

It should be noted that for the $\widetilde{E}$ and $\widetilde{B}$ fields in (23, 24) we used the same definition and notation as given by ([42]); this definition for the scalar components seems somewhat natural as it follows directly from the application of the spin raising-spin lowering operation to polarization data. More commonly, in the CMB literature the $E$ and $B$ random fields are defined instead as

$$
\widehat{E}(\hat{\gamma})=\sum_{l m} a_{l m ; E} Y_{l m}(\hat{\gamma}), \widehat{B}(\hat{\gamma})=\sum_{l m} a_{l m ; B} Y_{l m}(\hat{\gamma})
$$

i.e., these fields differ by a normalization factor $\sqrt{(l+2) ! /(l-2) !}$ multiplying the random spherical harmonic coefficients $\left\{a_{l m ; E}, a_{l m ; B}\right\}$. It is indeed possible to obtain needlet approximations of these maps, by focussing on

$$
\begin{aligned}
& \widehat{E}^{*}(\hat{\gamma}) \equiv-\frac{1}{2}\left\{\sum_{j k}\left[\beta_{j k ; 2}^{*} \widehat{\varphi}_{j k}(\hat{\gamma})+\overline{\beta_{j k ; 2}^{*} \widehat{\varphi}_{j k}}(\hat{\gamma})\right]\right\}, \\
& \widehat{B}^{*}(\hat{\gamma}) \equiv \frac{i}{2} \sum_{j k}\left[\beta_{j k ; 2}^{*} \widehat{\varphi}_{j k}(\hat{\gamma})-\overline{\beta_{j k ; 2}^{*} \widehat{\varphi}_{j k}}(\hat{\gamma})\right],
\end{aligned}
$$


where

$$
\widehat{\varphi}_{j k}(\hat{\gamma}) \stackrel{\text { def }}{=} \sqrt{\lambda_{j k}} \sum_{\ell m} b\left(\frac{\ell}{B^{j}}\right)\left\{Y_{\ell m}(\hat{\gamma})\right\}\left\{\overline{{ }_{2} Y_{\ell m}}\left(\xi_{j k}\right)\right\}
$$

It should be noted however that $\widehat{E}(\hat{\gamma}), \widehat{B}(\hat{\gamma})$ do not follow trivially by application of the spin raising - spin lowering operators to polarization data; likewise, and as opposed to (20), (27) is not the outcome of differentiation by $\partial(\bar{\partial})$ on spin needlets, so that its properties are less clear. (Because of (21), (20) is well-localized by the results of [34].)

The results in the present subsection suggest that spin needlets may allow for new statistical techniques when dealing with map reconstruction, even besides those allowed by wavelet methods in the scalar case. More generally, due to the poor localization properties of standard spherical harmonics, in the presence of masked regions and instrumental noise the construction of $E$ and $B$ from the coefficients $\left\{\widetilde{a}_{l m ; 2}\right\}$ (see (15)) may not ensure satisfactory results (recall $\left\{\widetilde{a}_{l m ; 2}\right\}$ need not be close to $\left\{a_{l m ; 2}\right\}$ in any meaningful sense for partially observed polarization maps). On the other hand, in view of (16) the coefficients $\left\{\widetilde{\beta}_{j k}\right\}$, are much less affected by masked regions, despite the fact that these quantities can themselves be viewed as linear combinations of the spherical harmonic coefficients. This suggests that spin needlet coefficients may be extremely useful when attempting to build optimal polarization maps; for instance, it seems natural to suggest the implementation of Internal Linear Combination techniques based on wavelet coefficients (as done in the scalar case by [26]), without the need to go through scalar maps as a first step. These developments are left for future work.

\section{Estimation of the angular power spectra $C_{l}^{E E}, C_{l}^{B B}$}

We are now ready to address the estimation of the angular power spectra $C_{l}^{E E}, C_{l}^{B B}$ as separate quantities, which is clearly much more meaningful from a physical point of view. For this purpose, it suffices to write

$$
\begin{aligned}
E_{j}(\hat{\gamma}) & =-\frac{1}{2} \sum_{l m} b^{2}\left(\frac{l}{B^{j}}\right)\left(a_{l m ; 2}+\bar{a}_{l-m ; 2}\right) Y_{l m}(\hat{\gamma}) \sqrt{\frac{(l+2) !}{(l-2) !}} \\
& =\sum_{l m} b^{2}\left(\frac{l}{B^{j}}\right) a_{l m ; E} Y_{l m}(\hat{\gamma}) \sqrt{\frac{(l+2) !}{(l-2) !}}
\end{aligned}
$$


and likewise

$$
B_{j}(\hat{\gamma})=\sum_{l m} b^{2}\left(\frac{l}{B^{j}}\right) a_{l m ; B} Y_{\ell m}(\hat{\gamma}) \sqrt{\frac{(l+2) !}{(l-2) !}} .
$$

Clearly

$$
\begin{gathered}
<\int_{S^{2}} E_{j}^{2}(\hat{\gamma}) d \widehat{\gamma}>=\int_{S^{2}}<E_{j}^{2}(\hat{\gamma})>d \widehat{\gamma} \\
=\sum_{l} b^{2}\left(\frac{l}{B^{j}}\right) C_{l}^{E E} \frac{(l+2) !}{(l-2) !} \stackrel{d e f}{=} \widetilde{\Gamma}_{j}^{E E}, \\
<\int_{S^{2}} B_{j}^{2}(\hat{\gamma}) d \widehat{\gamma}>=\sum_{l} b^{2}\left(\frac{l}{B^{j}}\right) C_{l}^{B B} \frac{(l+2) !}{(l-2) !}=\widetilde{\Gamma}_{j}^{B B} .
\end{gathered}
$$

In the presence of a mask, it is then natural to suggest the following (unbiased) estimator:

$$
\widetilde{C}_{j}^{E E}=\frac{\int_{S^{2} / M}\left(\widetilde{E}_{j}^{*}\right)^{2}(\hat{\gamma}) d \widehat{\gamma}}{\int_{S^{2} / M} d \widehat{\gamma}}, \widetilde{C}_{j}^{B B}=\frac{\int_{S^{2} / M}\left(\widetilde{B}_{j}^{*}(\hat{\gamma})\right)^{2} d \widehat{\gamma}}{\int_{S^{2} / M} d \widehat{\gamma}} ;
$$

here, the denominator is just a sky-fraction normalizing factor, whereas the integrals can be easily approximated by sums in pixel spaces. It is obvious that these estimators are unbiased for $\widetilde{\Gamma}_{j}^{E E}, \widetilde{\Gamma}_{j}^{B B} ;$ likewise, one might focus on

$$
\widehat{C}_{j}^{E E}=\frac{\int_{S^{2} / M}\left(\widehat{E}_{j}^{*}(\hat{\gamma})\right)^{2} d \widehat{\gamma}}{\int_{S^{2} / M} d \widehat{\gamma}}, \widehat{C}_{j}^{B B}=\frac{\int_{S^{2} / M}\left(\widehat{B}_{j}^{*}(\hat{\gamma})\right)^{2} d \widehat{\gamma}}{\int_{S^{2} / M} d \widehat{\gamma}}
$$

which are unbiased for the binned spectra $\sum_{l} b^{2}\left(l / B^{j}\right) C_{l}^{E E}, \sum_{l} b^{2}\left(l / B^{j}\right) C_{l}^{B B}$. A more complete set of properties and implementation on polarization data will be provided elsewhere.

\section{A COMPARISON WITH ALTERNATIVE CONSTRUCTIONS}

To compare our approach with possible alternative constructions (see also [44]), let us consider again the scalar fields of $\widehat{E}$ - and $\widehat{B}$-modes, which we recall can be written as

$$
\widehat{E}(\hat{\gamma}):=\sum_{l m} a_{l m ; E} Y_{l m}(\hat{\gamma}), \widehat{B}(\hat{\gamma}):=\sum_{l m} a_{l m ; B} Y_{l m}(\hat{\gamma})
$$

In the idealistic case of fully observed and noiseless maps for $F(\hat{\gamma})=\{Q(\hat{\gamma})+i U(\hat{\gamma})\}$, the coefficients $\left\{a_{l m ; E}, a_{l m ; B}\right\}$ could be exactly recovered and it would be natural to derive the scalar random needlet coefficients on $\widehat{E}(\hat{\gamma}), \widehat{B}(\hat{\gamma})$ as

$$
\beta_{j k}^{E}=\sqrt{\lambda_{j k}} \sum_{l m} b\left(\frac{l}{B^{j}}\right) a_{l m ; E} Y_{l m}\left(\xi_{j k}\right), \beta_{j k}^{B}=\sqrt{\lambda_{j k}} \sum_{l m} b\left(\frac{l}{B^{j}}\right) a_{l m ; B} Y_{l m}\left(\xi_{j k}\right) .
$$


In other words, one might propose to define a wavelet construction by deriving first the scalar maps, and then applying on them the standard procedures. The purpose of this Section is to compare this approach with the one we suggested earlier to show that they are not equivalent. More precisely, we shall address the question about the localization properties of (28), and investigate the effect of the presence of a masked region $M$.

Recall that for scalar fields, when $\xi_{j k}$ is "far enough" from $M$ we have the approximation

$$
\begin{aligned}
\beta_{j k}^{E} & =\sqrt{\lambda_{j k}} \sum_{l m} b\left(\frac{l}{B^{j}}\right) a_{l m ; E} Y_{l m}\left(\xi_{j k}\right) \\
& =\int_{S^{2}} \widehat{E}(\hat{\gamma}) \psi_{j k}(\hat{\gamma}) d \hat{\gamma} \simeq \int_{S^{2} / M} \widehat{E}(\hat{\gamma}) \psi_{j k}(\hat{\gamma}) d \hat{\gamma}=\widetilde{\beta}_{j k}^{E} .
\end{aligned}
$$

In the presence of a masked region we obtain for instance

$$
\begin{aligned}
& \widetilde{\widetilde{\beta}}_{j k}^{E}=\sqrt{\lambda_{j k}} \sum_{l m} b\left(\frac{l}{B^{j}}\right) \widetilde{a}_{l m ; E} Y_{l m}\left(\xi_{j k}\right), \\
& \widetilde{a}_{l m}^{E}=-\frac{1}{2}\left\{\int_{S^{2} / M}\left[F(\hat{\gamma})\left(\overline{{ }_{2} Y_{l m}}(\hat{\gamma})\right)+\bar{F}(\widehat{\gamma})\left(\overline{{ }_{-2} Y_{l m}}(\hat{\gamma})\right)\right] d \hat{\gamma}\right\} .
\end{aligned}
$$

Now note that

$$
\begin{gathered}
\widetilde{\widetilde{\beta}}_{j k}^{E}-\beta_{j k}^{E}=-\sqrt{\lambda_{j k}} \sum_{l m} b\left(\frac{l}{B^{j}}\right) \frac{1}{2}\left\{\widetilde{a}_{l m}^{E}-a_{l m}^{E}\right\} Y_{l m}\left(\xi_{j k}\right) \\
=-\frac{1}{2} \sqrt{\lambda_{j k}} \sum_{l m} b\left(\frac{l}{B^{j}}\right)\left\{\int_{M}\left[F(\hat{\gamma})\left(\overline{{ }_{2} Y_{l m}}(\hat{\gamma})\right)+\bar{F}(\hat{\gamma})\left(\overline{{ }_{-2} Y_{l m}}(\hat{\gamma})\right)\right] d \hat{\gamma}\right\} Y_{l m}\left(\xi_{j k}\right) \\
=-\frac{1}{2} \int_{M}\left[F(\hat{\gamma}) \bar{\chi}_{j k ; 2}(\hat{\gamma})+\bar{F}(\hat{\gamma}) \bar{\chi}_{j k ;-2}(\hat{\gamma})\right] d \hat{\gamma},
\end{gathered}
$$

where

$$
\begin{gathered}
\left.\chi_{j k ; 2}(\hat{\gamma})=\sqrt{\lambda_{j k}} \sum_{l m} b\left(\frac{l}{B^{j}}\right){ }_{2} Y_{l m}(\hat{\gamma})\right) \overline{Y_{l m}}\left(\xi_{j k}\right), \\
\chi_{j k ;-2}(\hat{\gamma})=\sqrt{\lambda_{j k}} \sum_{l m} b\left(\frac{l}{B^{j}}\right)\left({ }_{-2} Y_{l m}(\hat{\gamma})\right) \overline{Y_{l m}}\left(\xi_{j k}\right) .
\end{gathered}
$$

Now the properties of (30), (31) are still unknown and lack a rigorous mathematical exploration. Indeed the function $\sum_{m}\left(\overline{{ }_{2} Y_{l m}(\hat{\gamma})}\right) Y_{l m}\left(\xi_{j k}\right)$ does not represent any form of projector on a proper subspace, as it is the case for the corresponding expression $\sum_{m}\left(\overline{Y_{l m}(\hat{\gamma})}\right) Y_{l m}\left(\xi_{j k}\right)$ for the standard needlet construction in the scalar case. In fact looking at figure 6 (compare with figure 2 for the spin needlets) we see that the shape of $\left|\chi_{j k ; 2}(\hat{\gamma})\right|$ is such that at the origin the function has a zero, rather than a maximum, and 

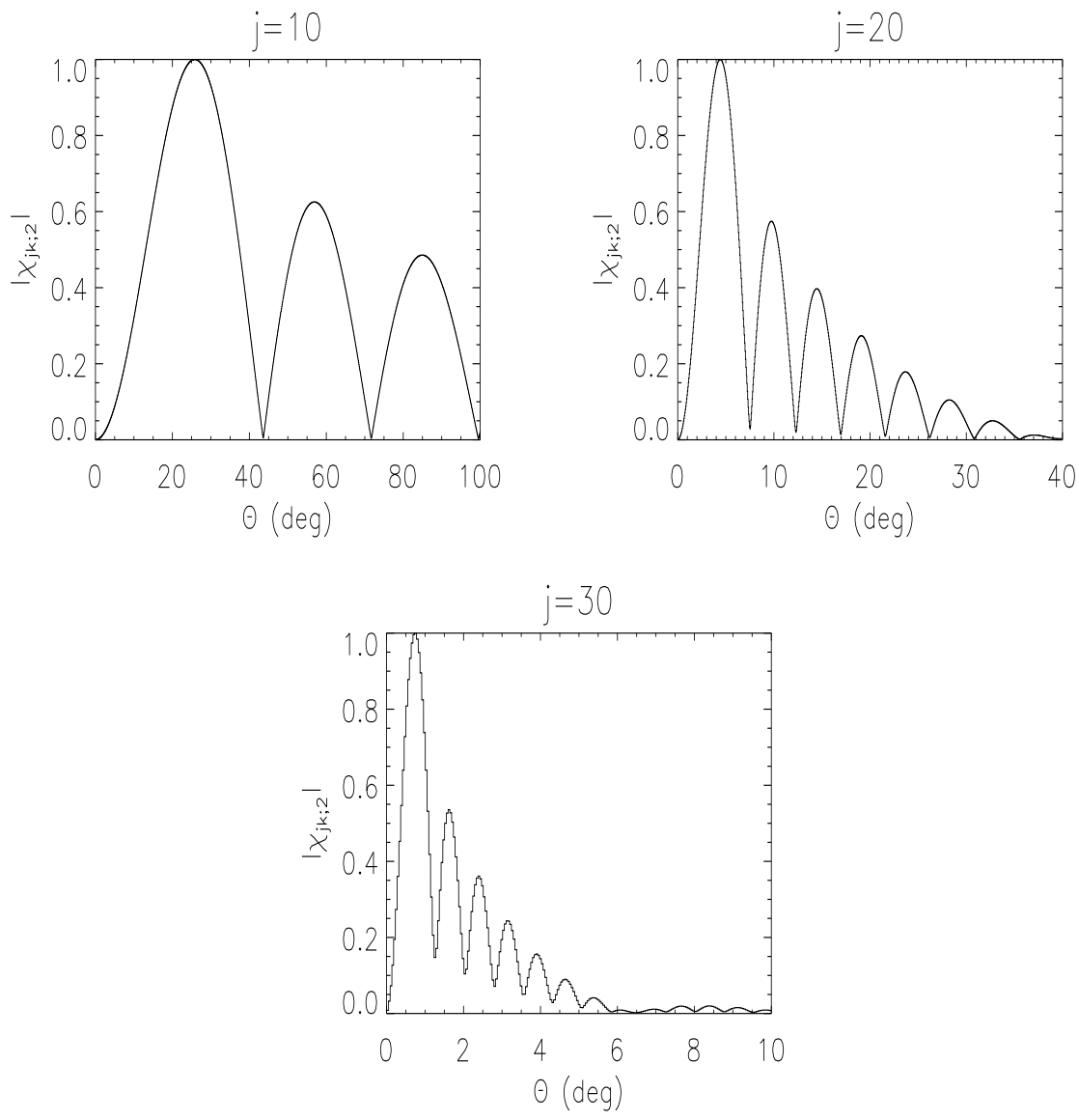

FIG. 6: The modulus of $\chi_{j k ; 2}(\hat{\gamma})$ for $B=1.2$ and $j=10$ (left plot), $j=20$ (right plot) and $j=30$ (lower plot). The angle $\theta$ is the distance from the center point.

then increases away from the center. In other words, while spin needlets enjoy a number of properties which are analogous to those in the scalar case (good approximation properties in the presence of a mask (see (16) above), tight frame property, asymptotic uncorrelation), these properties are not in general known to be shared by constructing wavelets on the scalar random fields $\widehat{E}, \widehat{B}$ after implementing the spherical harmonic transforms, in the presence of masked regions.

We believe these remarks provide a clear rationale for our construction of spin needlets, which are directly embedded into the geometric structure of polarization random fields. 


\section{Acknowledgements}

We are grateful to a referee for a careful reading of the manuscript and useful suggestions, and to Xiaohong Lan for many useful suggestions on a previous draft. FKH is also grateful for an OYI grant from the Research Council of Norway.

[1] Hinshaw, G., Weiland, J. L., Hill, R. S., Odegard, N., Larson, D., Bennett, C. L., Dunkley, J., Gold, B., Greason, M. R., Jarosik, N., Komatsu, E., Nolta, M. R., Page, L., Spergel, D. N., Wollack, E., Halpern, M., Kogut, A., Limon, M., Meyer, S. S., Tucker, G. S., Wright, E. L. (2008) Five-Year Wilkinson Microwave Anisotropy Probe (WMAP) Observations: Data Processing, Sky Maps, and Basic Results eprint arXiv:0803.0732

[2] Laureijs, R. J.(On Behalf Of The Planck Collaboration) (2007), Polarization Maps at CMB Frequencies from Planck, EAS Publications Series, Volume 23, 2007, pp.247-254

[3] Antoine, J.-P. and Vandergheynst, P. (1999) Wavelets on the Sphere: a GroupTheoretic Approach, Applied and Computational Harmonic Analysis, 7, pp. 262-291

[4] Antoine J.-P., Demanet L., Jacques L., Vandergheynst P. (2002) Wavelets on the sphere: implementation and approximations, Applied and Computational Harmonic Analysis, $13,177-200$.

[5] Antoine, J.-P. and Vandergheynst, P. (2007), Wavelets on the Sphere and Other Conic Sections, Journal of Fourier Analysis and its Applications, 13, 369-386

[6] Gorski, K. M., Lilje P. B. (2006), "Foreground Subtraction of Cosmic Microwave Background Maps using WI-FIT (Wavelet based hIgh resolution Fitting of Internal Templates)", Astrophysical J., $648,784-796$.

[7] Vielva, P., Martínez-González, E., Gallegos, J. E., Toffolatti, L., Sanz, J. L. (2003) Point Source Detection Using the Spherical Mexican Hat Wavelet on Simulated All-Sky Planck Maps, Monthly Notice of the Royal Astronomical Society, Volume 344, Issue 1, pp. 89-104.

[8] Sanz J.L., Herranz D., Lopez-Caniego M., Argueso F. (2006) Wavelets on the sphere. Application to the detection problem, Proceedings of the 14th European Signal Processing Conference, Eds. F. Gini and E.E. Kuruoglu. 
[9] Vielva P., Martinez-Gonzalez E., Barreiro B., Sanz J., Cayon L. (2004) Detection of non-Gaussianity in the WMAP first year data using spherical wavelets, Astrophysical J., Volume 609, pp. 22-34.

[10] Cabella, P., Hansen, F.K., Marinucci, D., Pagano, D. and Vittorio, N. (2004) Search for non-Gaussianity in Pixel, Harmonic, and Wavelet Space: Compared and Combined. Physical Review D 69 06300\%.

[11] Jin J., Starck J.-L., Donoho D. L., Aghanim N., Forni, O. Cosmological non-Gaussian signature detection: comparing performance of different statistical tests. EURASIP J. Appl. Signal Process., 2470-2485.

[12] Cruz, M., Cayon, L., Martinez-Gonzalez, E., Vielva, P., Jin, J., (2007) The nonGaussian Cold Spot in the 3-year WMAP Data, Astrophysical Journal 655, 11-20

[13] Cruz, M., Cayon, L., Martinez-Gonzalez, E., Vielva, P., (2006) The non-Gaussian Cold Spot in WMAP: Significance, Morphology and Foreground Contribution, Monthly Notices of the Royal Astronomical Society 369, 57-67

[14] Moudden Y., Cardoso J.-F., Starck J.-L., Delabrouille J. (2005), Blind component separation in wavelet space.Application to CMB analysis, EURASIP J.Appl.Signal Process.\} 15, pp. 2437-2454

[15] McEwen J. D., Vielva P., Hobson M. P., Martinez-Gonzalez E., Lasenby A. N. (2007) Detection of the integrated Sachs-Wolfe effect and corresponding dark energy constraints made with directional spherical wavelets, Monthly Notices Roy. Astronom. Soc., 376 (3), 1211-1226.

[16] McEwen J.D., Hobson M.P., Lasenby A.N., Mortlock, D.J. (2006) A highsignificance detection of non-Gaussianity in the WMAP 3-year data using directional spherical wavelets, Monthly Notices Roy. Astronom. Soc., 371, Issue 123002, L50-L54.

[17] Wiaux I., McEwen J. D., Vandergheynst P., Blanc O. (2008) Exact reconstruction with directional wavelets on the sphere, Monthly Notices of the Royal Astronomical Society, Volume 388, Issue 2, pp. 770-788.

[18] Narcowich, F.J., Petrushev, P. and Ward, J.D. (2006a) Localized Tight Frames on Spheres, SIAM Journal of Mathematical Analysis 38, 2, 574-594

[19] Narcowich, F.J., Petrushev, P. and Ward, J.D. (2006b) Decomposition of Besov and Triebel-Lizorkin Spaces on the Sphere, Journal of Functional Analysis 238, 2, 530-564 
[20] Baldi, P., Kerkyacharian, G., Marinucci, D. and Picard, D. (2006) Asymptotics for Spherical Needlets, Annals of Statistics, in press, arxiv:math/0606599

[21] Baldi, P., Kerkyacharian, G. Marinucci, D. and Picard, D. (2007) Subsampling Needlet Coefficients on the Sphere, Bernoulli, in press, arxiv 0706.4169

[22] Pietrobon, D., Balbi, A., Marinucci, D. (2006) Integrated Sachs-Wolfe Effect from the Cross Correlation of WMAP3 Year and the NRAO VLA Sky Survey Data: New Results and Constraints on Dark Energy, Physical Review D, 74, 043524

[23] Marinucci, D., Pietrobon, D., Balbi, A., Baldi, P., Cabella, P., Kerkyacharian, G., Natoli, P., Picard, D., Vittorio, N. (2008) Spherical Needlets for CMB Data Analysis, Monthly Notices of the Royal Astronomical Society, Vol. 383, 539-545 arxiv:0707/0844

[24] Guilloux, F., Fay, G., Cardoso, J.-F. (2007) Practical Wavelet Design on the Sphere, arxiv 0706.2598

[25] Lan, X., Marinucci, D. (2008) The Needlets Bispectrum, Electronic Journal of Statistics, 2, 332-367, arxiv: 0802.4020

[26] Delabrouille, J., Cardoso, J.-F. , Le Jeune, M. , Betoule, M., Fay, G. ,Guilloux, F. (2008) A full sky, low foreground, high resolution CMB map from WMAP, arxiv 0807.0773

[27] Fay, G., Guilloux, F. , Betoule, M. , Cardoso, J.-F. , Delabrouille, J. , Le Jeune, M. (2008), CMB power spectrum estimation using wavelets, arxiv 0807.1113

[28] Fay, G. and Guilloux, F. (2008) Consistency of a needlet spectral estimator on the sphere, arxiv: 0807.2162

[29] Geller, D. and Mayeli, A. (2008) Continuous wavelets on manifolds, accepted by and published on the website of Math. Z., 33 pages, also on ArXiv.

[30] Geller, D. and Mayeli, A. (2008) Nearly tight frames and space-frequency analysis on compact manifolds, accepted by and published on the website of Math. Z., 30 pages, also on ArXiv.

[31] Geller, D. and Mayeli, A. (2007) Besov Spaces and Frames on Compact Manifolds arxiv: 0709.2452

[32] Lan, X., Marinucci, D. (2008) On the dependence structure of wavelet coefficients for spherical random fields, submitted, arxiv: 0805.4154

[33] Mayeli, A. (2008) Asymptotic Uncorrelation for Mexican Needlets, submitted, arxiv: 0806.3009 
[34] Geller, D. and Marinucci, D. (2008) Spin Wavelets on the sphere, submitted, arxiv: 0811.2935.

[35] Hernández, E., Weiss, G. (1996) A first course on wavelets. Studies in Advanced Mathematics. CRC Press.

[36] Baldi, P., Kerkyacharian, G., Marinucci, D., Picard, D. (2008) Adaptive density estimation for directional data using needlets, 0807.5059

[37] Doroshkevich, A.G., Naselsky, P.D., Verkhodanov, O.V. , Novikov, D.I., Turchaninov, V.I., Novikov, I.D., Christensen, P.R., Chiang, L.-Y. (2005) Gauss-Legendre Sky Pixelization (GLESP) for CMB Maps, International Journal of Modern Physics D 14, 275, arxiv:astro-ph/0305537

[38] Gorski, K. M. , Hivon, E., Banday, A. J., Wandelt, B. D., Hansen, F. K., Reinecke, M., Bartelman, M., (2005) HEALPix - a Framework for High Resolution Discretization, and Fast Analysis of Data Distributed on the Sphere, Astrophysical Journal 622, 759-7r1, arxiv:astro-ph/0409513

[39] Seljak, U. and Zaldarriaga, M. (1997) An all-sky analysis of polarization in the microwave background, Physical Review D, Vol. 55, N.4, 1830-1840

[40] Kamionkowski, M., Kosowsky, A., Stebbins, A. (1997) Statistics of cosmic microwave background polarization, Physical Review D, Volume 55, Issue 12, pp.7368-7388

[41] Pietrobon, D., Amblard, A., Balbi, A., Cabella, P., Cooray, A., Marinucci, D. (2008) Needlet Detection of Features in WMAP CMB Sky and the Impact on Anisotropies and Hemispherical Asymmetries, Physical Review D, in press, eprint arXiv:0809.0010

[42] Wiaux, Y., Jacques, L., Vandergheynst, P. (2007) Fast spin \pm 2 spherical harmonics and applications in cosmology, Journal of Computational Physics, v. 226, iss. 2, p. 2359-2371

[43] Donoho, D. L. , Johnstone, I. M.; Kerkyacharian, G., Picard, D. (1997) Density estimation by wavelet thresholding. Ann. Statist. 24 (1996), no. 2, 508-539.

[44] Cabella, P., Natoli, P.; Silk, J. (2007) Constraints on CPT violation from WMAP three year polarization data: a wavelet analysis. Physical Review D, 76, 123014, eprint arXiv:0705.0810 


\section{APPENDIX: SPIN SPHERICAL HARMONICS}

In this Appendix, we review a few basic facts about the analysis of polarization data. Our review will be quite informal, and we will follow mainly the formalism by [39]; we refer also to [40] for an equivalent approach and a much more detailed treatment, see also [42]. All the material discussed here is absolutely standard, and it is reported just for completeness.

A standard route for the expansion of polarization random fields is based on spin spherical harmonics (see for instance $([39],[42]))$. Consider the standard representation of the group of rotations $S O(3)$ by means of the Wigner's $D$-matrices, with elements

$$
\begin{gathered}
D_{m n}^{l}(\varphi, \vartheta, \chi)=e^{-i m \varphi} d_{m n}^{l}(\vartheta) e^{-i n \chi} \\
d_{m n}^{l}(\vartheta)=\sum_{t=0 \vee(m-n)}^{(l+m) \wedge(l-n)} \frac{(-1)^{t}[(l+m) !(l-m) !(l+n) !(l-n) !]^{1 / 2}}{(l+m-t) !(l-n-t) ! t !(t+n-m) !}\left(\cos \frac{\vartheta}{2}\right)^{2 l+m-n-2 t}\left(\sin \frac{\vartheta}{2}\right)^{2 t+n-m},
\end{gathered}
$$

where we recall that

$$
Y_{l m}(\vartheta, \varphi) \equiv \sqrt{\frac{2 l+1}{4 \pi}} D_{m 0}^{l}(\varphi, \vartheta, 0)
$$

The spherical harmonics of spin $n$ can then similarly be defined as

$$
\begin{aligned}
{ }_{n} Y_{l m}(\vartheta, \varphi) & \equiv(-1)^{n} \sqrt{\frac{2 l+1}{4 \pi}} D_{m(-n)}^{l *}(\varphi, \vartheta, 0) \\
& =(-1)^{n} \sqrt{\frac{2 l+1}{4 \pi}} d_{m(-n)}^{l}(\vartheta) e^{i m \varphi} .
\end{aligned}
$$

An important property we shall repeatedly use is

$$
\sum_{m=-l}^{l}\left\{{ }_{n} Y_{l, m}(\vartheta, \varphi)\right\}\left\{{ }_{n} \overline{Y_{l, m}(\vartheta, \varphi)}\right\}=\frac{2 l+1}{4 \pi},
$$

see for instance [34], Theorem 2.8.

Under a rotation around the tangent plane at the point $(\vartheta, \varphi),{ }_{n} Y_{l m}(\vartheta, \varphi)$ transforms into $e^{-i n \chi} Y_{l m}(\vartheta, \varphi)$, i.e. as a spin $n$ function. Spin $n$ functions can be defined iteratively by means of the spin raising and lowering operators

$$
\begin{aligned}
& \partial\left({ }_{n} G(\vartheta, \varphi)\right):=\left[-\sin ^{n} \vartheta\left(\frac{\partial}{\partial \vartheta}+\frac{i}{\sin \vartheta} \frac{\partial}{\partial \varphi}\right) \sin ^{-n} \vartheta\right]\left({ }_{n} G(\vartheta, \varphi)\right) \\
& \bar{\partial}\left({ }_{n} G(\vartheta, \varphi)\right):=\left[-\sin ^{-n} \vartheta\left(\frac{\partial}{\partial \vartheta}-\frac{i}{\sin \vartheta} \frac{\partial}{\partial \varphi}\right) \sin ^{n} \vartheta\right]\left({ }_{n} G(\vartheta, \varphi)\right) .
\end{aligned}
$$

We have for instance

$$
\left.\partial{ }_{n} Y_{l m}(\vartheta, \varphi)\right)=[(l-n)(l+n+1)]^{1 / 2}\left({ }_{n+1} Y_{l m}(\vartheta, \varphi)\right)
$$


whence

$$
(\partial)^{2}\left(Y_{l m}(\vartheta, \varphi)\right)=[l(l+1)]^{1 / 2}[(l-1)(l+2)]^{1 / 2}\left({ }_{2} Y_{l m}(\vartheta, \varphi)\right),
$$

which generalizes iteratively to (for $0 \leq n \leq l$ )

$$
{ }_{n} Y_{l m}(\vartheta, \varphi)=\left[\frac{(l-n) !}{(l+n) !}\right]^{1 / 2}(\partial)^{n}\left(Y_{l m}(\vartheta, \varphi)\right),
$$

and (for $0 \geq n \geq-l$ )

$$
{ }_{n} Y_{l m}(\vartheta, \varphi)=\left[\frac{(l+n) !}{(l-n) !}\right]^{1 / 2}(-1)^{n}(\bar{\partial})^{n}\left(Y_{l m}(\vartheta, \varphi)\right) .
$$

More explicitly

$$
\begin{gathered}
{ }_{2} Y_{l m}(\vartheta, \varphi)=\left[\frac{(l-2) !}{(l+2) !}\right]^{1 / 2}\left[-\sin \vartheta\left(\frac{\partial}{\partial \vartheta}+\frac{i}{\sin \vartheta} \frac{\partial}{\partial \varphi}\right) \sin ^{-1} \vartheta\right]\left[-\left(\frac{\partial}{\partial \vartheta}+\frac{i}{\sin \vartheta} \frac{\partial}{\partial \varphi}\right)\right] Y_{l m}(\vartheta, \varphi) \\
=\left[\frac{(l-2) !}{(l+2) !}\right]^{1 / 2}\left[-\sin \vartheta\left(\frac{\partial}{\partial \vartheta}+\frac{i}{\sin \vartheta} \frac{\partial}{\partial \varphi}\right) \sin ^{-1} \vartheta\right]\left[\frac{m}{\sin \vartheta} Y_{l m}(\vartheta, \varphi)-\frac{\partial}{\partial \vartheta} Y_{l m}(\vartheta, \varphi)\right] .
\end{gathered}
$$

Simple computations yield

$$
\sqrt{\frac{(l+2) !}{(l-2) !}}\left\{{ }_{ \pm 2} Y_{l m}\right\}=\left[\frac{m^{2}}{\sin ^{2} \vartheta}-\frac{2 m}{\sin \vartheta}+2 m \frac{\cot \vartheta}{\sin \vartheta}-\cot \vartheta \frac{\partial}{\partial \vartheta}+\frac{\partial^{2}}{\partial \vartheta^{2}}\right] Y_{l m} .
$$

We can then introduce the $E$ and $B$ scalar components, whose random coefficients coefficients can be obtained (see ([39], [42])) evaluating the spin 2 transforms

$$
{ }_{ \pm 2} \widehat{Q}_{l m}:=\int_{S^{2}} Q(x)\left\{{ }_{ \pm 2} Y_{l m}(x)\right\} d x,{ }_{ \pm 2} \widehat{U}_{l m}:=\int_{S^{2}} U(x)\left\{{ }_{ \pm 2} Y_{l m}(x)\right\} d x,
$$

and then proceeding to evaluate the coefficients

$$
\begin{aligned}
& a_{l m}^{E}=-\frac{1}{2}\left[\left({ }_{2} \widehat{Q}_{l m}+i_{2} \widehat{U}_{l m}\right)+\left({ }_{-2} \widehat{Q}_{l m}-i_{-2} \widehat{U}_{l m}\right)\right], \\
& a_{l m}^{B}=\frac{i}{2}\left[\left({ }_{2} \widehat{Q}_{l m}+i_{2} \widehat{U}_{l m}\right)-\left({ }_{-2} \widehat{Q}_{l m}-i_{-2} \widehat{U}_{l m}\right)\right] .
\end{aligned}
$$

